\title{
Carbon and oxygen isotope ratios in starburst galaxies: New data from NGC 253 and Mrk 231 and their implications
}

\author{
C. Henkel ${ }^{1,2}$, H. Asiri ${ }^{2}$, Y. Ao ${ }^{1,3,4}$, S. Aalto ${ }^{5}$, A. L. R. Danielson ${ }^{6}$, P. P. Papadopoulos ${ }^{7,8}$, S. García-Burillo ${ }^{9}$, \\ R. Aladro ${ }^{10}$, C. M. V. Impellizzeri ${ }^{10}$, R. Mauersberger ${ }^{10}$, S. Martín ${ }^{11}$, and N. Harada ${ }^{1}$
}

1 Max-Planck-Institut für Radioastronomie, Auf dem Hügel 69, 53121 Bonn, Germany

e-mail: chenkel@mpifr-bonn.mpg.de

2 Astronomy Department, Faculty of Science, King Abdulaziz University, PO Box 80203, 21589 Jeddah, Saudi Arabia

3 Purple Mountain Observatory, Chinese Academy of Sciences, 21008 Nanjing, PR China

${ }^{4}$ National Astronomical Observatory of Japan, 2-21-1 Osawa, Mitaka, 181-8588 Tokyo, Japan

5 Dept. of Earth and Space Sciences, Chalmers University of Technology, Onsala Observatory, 43994 Onsala, Sweden

${ }^{6}$ Institute for Computational Cosmology, Dept. of Physics, Durham University, South Road, Durham DH1 3LE, UK

7 School of Physics, Cardiff University, Queens Building, The Parade, Cardiff CF24 3AA, UK

${ }^{8}$ European Southern Observatory, Karl-Schwarzschild-Strasse 2, 85748 Garching, Germany

9 Observatorio Astronómico Nacional (OAN) - Observatorio de Madrid, Alfonso XII, 3, 28014 Madrid, Spain

${ }_{10}$ European Southern Observatory, Avda. Alonso de Córdova 3107, Vitacura, Casilla 19001, Santiago, Chile

11 Institute de Radioastronomie Millimétrique, rue de la Piscine 300, 38406 Saint-Martin d'Hères, France

Received 31 October 2013 / Accepted 18 February 2014

\begin{abstract}
Carbon and oxygen isotope ratios are excellent measures of nuclear processing, but few such data have been taken toward extragalactic targets so far. Therefore, using the IRAM 30-m telescope, CN and CO isotopologues have been measured toward the nearby starburst galaxy NGC 253 and the prototypical ultraluminous infrared galaxy Mrk 231. Toward the center of NGC 253 , the $\mathrm{CN}$ and ${ }^{13} \mathrm{CN}$ $N=1 \rightarrow 0$ lines indicate no significant deviations from expected local thermodynamical equilibrium after accounting for moderate saturation effects (10 and 25\%) in the two detected spectral components of the main species. Including calibration uncertainties, which dominate the error budget, the ${ }^{12} \mathrm{C} /{ }^{13} \mathrm{C}$ ratio becomes $40 \pm 10$. This is larger than the ratio in the central molecular zone of the Galaxy, suggesting a higher infall rate of poorly processed gas toward the central region. Assuming that the ratio also holds for the $\mathrm{CO}$ emitting gas, this yields ${ }^{16} \mathrm{O} /{ }^{18} \mathrm{O}=145 \pm 36$ and ${ }^{16} \mathrm{O} /{ }^{17} \mathrm{O}=1290 \pm 365$ and a ${ }^{32} \mathrm{~S} /{ }^{34} \mathrm{~S}$ ratio close to the one measured for the local interstellar medium (20-25). No indication of vibrationally excited $\mathrm{CN}$ is found in the lower frequency fine structure components of the $N=1 \rightarrow 0$ and $2 \rightarrow 1$ transitions at rms noise levels of 3 and $4 \mathrm{mK}(15$ and $20 \mathrm{mJy})$ in $8.5 \mathrm{~km} \mathrm{~s}^{-1}$ wide channels. Peak line intensity ratios between NGC 253 and Mrk 231 are $\sim 100$ for ${ }^{12} \mathrm{C}^{16} \mathrm{O}$ and ${ }^{12} \mathrm{C}^{18} \mathrm{O} J=1 \rightarrow 0$, while the ratio for ${ }^{13} \mathrm{C}^{16} \mathrm{O} J=1 \rightarrow 0$ is $\sim 250$. This and similar ${ }^{13} \mathrm{CO}$ and $\mathrm{C}^{18} \mathrm{O}$ line intensities in the $J=1 \rightarrow 0$ and $2 \rightarrow 1$ transitions of Mrk 231 suggest ${ }^{12} \mathrm{C} /{ }^{13} \mathrm{C} \sim 100$ and ${ }^{16} \mathrm{O} /{ }^{18} \mathrm{O} \sim 100$, in agreement with values obtained for the less evolved ultraluminous merger Arp 220. Also, when accounting for other (scarcely available) extragalactic data, ${ }^{12} \mathrm{C} /{ }^{13} \mathrm{C}$ ratios appear to vary over a full order of magnitude, from $>100$ in ultraluminous high redshift galaxies to $\sim 100$ in more local such galaxies to 40 in weaker starbursts that are not undergoing a large scale merger to 25 in the central molecular zone of the Milky Way. With ${ }^{12} \mathrm{C}$ being predominantly synthesized in massive stars, while ${ }^{13} \mathrm{C}$ is mostly ejected by longer lived lower mass stars at later times, this is qualitatively consistent with our results of decreasing carbon isotope ratios with time and rising metallicity. It is emphasized, however, that both infall of poorly processed material, initiating a nuclear starburst, and the ejecta from newly formed massive stars (in particular in the case of a top-heavy stellar initial mass function) can raise the carbon isotope ratio for a limited amount of time.
\end{abstract}

Key words. galaxies: starburst - galaxies: abundances - galaxies: ISM - nuclear reactions, nucleosynthesis, abundances galaxies: individual: NGC 253 - galaxies: individual: Mrk 231

\section{Introduction}

When studying stellar nucleosynthesis and chemical enrichment, it is difficult to optically distinguish isotopes of a given element, since their atomic lines are blended. However, microwave lines from rare isotopic substitutions of a given molecular species, so-called "isotopologues", are separated well from their parent molecule, typically by a few percent of their rest frequency. Thus, the frequencies of the main and rare species are close enough to be observed with the same technical equipment but without the problem of blending.

A few years ago, it became apparent (Wouterloot et al. 2008; Wang et al. 2009) that with respect to its composition, the metal-poor outer Galaxy does not provide a "bridge" between the solar neighborhood and the even more metal-poor Large Magellanic Cloud (LMC). This can be explained by the different age of the bulk of the stellar populations of the outer Galaxy and the LMC and can be exemplified by one of the most thoroughly studied isotope ratios, that of carbon. The two stable isotopes, ${ }^{12} \mathrm{C}$ and ${ }^{13} \mathrm{C}$, have been measured throughout the Galaxy, in prominent star forming regions of the LMC, and in a large number of stellar objects (e.g., Milam et al. 2005; Wang et al. 2009; Abia et al. 2012; Mikolaitis et al. 2012). The ${ }^{12} \mathrm{C} /{ }^{13} \mathrm{C}$ ratio is a measure of "primary" versus "secondary" processing. ${ }^{12} \mathrm{C}$ is produced on rapid timescales primarily via He burning in massive stars. ${ }^{13} \mathrm{C}$ is mainly produced via $\mathrm{CNO}$ 
processing of ${ }^{12} \mathrm{C}$ seeds from earlier stellar generations. This occurs on a slower time scale during the red giant phase in low and intermediate mass stars or novae (for reviews, see Henkel et al. 1994; Wilson \& Rood 1994).

Previous observations have demonstrated that the ${ }^{12} \mathrm{C} /{ }^{13} \mathrm{C}$ ratio can vary strongly within the Galaxy (e.g., Henkel et al. 1985; Stahl et al. 1989; Wouterloot \& Brand 1996; Milam et al. 2005; Sheffer et al. 2007). In the outer Galaxy very high ratios of ${ }^{12} \mathrm{C} /{ }^{13} \mathrm{C}>100$ are found, and in the local interstellar medium ${ }^{12} \mathrm{C} /{ }^{13} \mathrm{C} \sim 70$, while in the inner Galactic disk and LMC ${ }^{12} \mathrm{C} /{ }^{13} \mathrm{C} \sim 50$. The solar system ratio is 89 . Within the framework of "biased infall" (e.g., Chiappini \& Matteucci 2001), the Galactic disk is slowly formed from inside out, which causes gradients in the abundances across the disk. The stellar ${ }^{13} \mathrm{C}$ ejecta, which reach the interstellar medium with a time delay, are less dominant in the young stellar disk of the outer Galaxy than in the inner Galaxy and the old stellar body of the LMC (see, e.g., Hodge 1989 for the star formation history of the LMC). The solar system ratio, referring to a younger more ${ }^{13} \mathrm{C}$ deficient disk, is therefore higher than is measured in the present local interstellar medium. Consistent with this idea, ${ }^{12} \mathrm{C} /{ }^{13} \mathrm{C}$ ratios are particularly low $(\sim 25)$ in the Galactic center region with its old bulge (e.g., Güsten et al. 1985), while inflowing or infalling gas from outside appears to be characterized by higher ratios (Riquelme et al. 2010). We note that this scenario also explains other isotope ratios based on differences in primary and secondary nucleosynthesis, such as for ${ }^{16} \mathrm{O}$ (a product of massive stars, $\gtrsim 8 M_{\odot}$ ) and ${ }^{17} \mathrm{O}$ (a product of lower mass stars), while ${ }^{18} \mathrm{O}$ is apparently synthesized most efficiently in metal-rich stars of high mass (e.g., Wouterloot et al. 2008).

With respect to isotope ratios, the extragalactic space beyond the Magellanic Clouds is almost unexplored and therefore very interesting to investigate (for previous pioneering efforts, see Aalto et al. 1991; Casoli et al. 1992; Henkel et al. 1993, 2010; Henkel \& Mauersberger 1993; Wang et al. 2004; Muller et al. 2006; Martín et al. 2010; González-Alfonso et al. 2012; Danielson et al. 2013). What ratios can be found when observing objects outside the Local Group of galaxies at low and high redshifts and in environments, which differ drastically from those in the Milky Way and the LMC? Is the Galaxy typical of its class or are its isotopic properties exceptional? And what kind of isotopic compositions can be expected in optical lines when trying to determine high precision redshifts and to constrain variations in physical constants through time and space (e.g., Levshakov et al. 2006)?

In the following we present and analyze new $\mathrm{CN}$ and CO data from the nearby prototypical starburst galaxy NGC 253 and the ultraluminous merger Mrk 231, in order to derive and to compare the carbon isotope ratios in these different environments. Section 2 describes observations and data reduction. Section 3 presents the $\mathrm{CN}$ and $\mathrm{CO}$ measurements and data analysis toward NGC 253, including carbon and oxygen isotope ratios and CN excitation temperatures. In Sect. 4 we discuss our data from Mrk 231 and provide a general overview of extragalactic carbon isotope determinations in targets beyond the Magellanic Clouds. Section 5 summarizes the main results.

\section{Observations}

The $\lambda \sim 3$ and $1.3 \mathrm{~mm}$ measurements toward NGC 253 were obtained with the IRAM 30-m telescope (project 078-12) at
Table 1. Observational parameters.

\begin{tabular}{ccccccc}
\hline \hline $\begin{array}{c}\text { Band } \\
(\mathrm{mm})\end{array}$ & $\begin{array}{c}v \\
(\mathrm{GHz})\end{array}$ & $\begin{array}{c}T_{\text {sys }} \\
(\mathrm{K})\end{array}$ & $\begin{array}{c}\theta_{\mathrm{b}} \\
\left({ }^{\prime \prime}\right)\end{array}$ & $f_{\mathrm{mb}}$ & $f_{\mathrm{fh}}$ & $\begin{array}{c}S / T_{\mathrm{mb}} \\
(\mathrm{Jy} / \mathrm{K})\end{array}$ \\
\hline 3 & 109.635 & 130 & 23 & 0.80 & 0.95 & 5.2 \\
3 & 113.365 & 240 & 22 & 0.80 & 0.95 & 5.1 \\
1.3 & 221.315 & 240 & 11 & 0.62 & 0.92 & 4.8 \\
1.3 & 225.045 & 260 & 11 & 0.62 & 0.92 & 4.8 \\
\hline
\end{tabular}

Notes. Receiver band $(\lambda \sim 3$ or $1.3 \mathrm{~mm})$, frequencies $(v)$, system temperatures $\left(T_{\text {sys }}\right)$ on an antenna temperature scale $\left(T_{\mathrm{A}}^{*}\right)$, full width to half power (FWHP) beam widths in units of arcseconds $\left(\theta_{\mathrm{b}}\right)$, and adopted main beam efficiencies $\left(f_{\mathrm{mb}}\right)$ and forward hemisphere efficiencies $\left(f_{\mathrm{fh}}\right)$ for the four $4 \mathrm{GHz}$ wide frequency intervals $(2 \times 8 \mathrm{GHz})$, simultaneously observed by the $30-\mathrm{m}$ telescope. The last column provides conversion factors from main beam brightness temperatures (in Kelvin) to flux density (Jansky) units.

Pico Veleta, Spain ${ }^{1}$, during August 5 and 6, 2012. Full width to half power beam widths (FWHPs) were about 22" and 11". The EMIR SiS receivers were employed with system temperatures of $T_{\text {sys }} \sim 130,240,240$, and $260 \mathrm{~K}$ at $109.6,113.4$ $(\lambda \sim 3 \mathrm{~mm}), 221.3$, and $225.0 \mathrm{GHz}(\lambda \sim 1.3 \mathrm{~mm})$ on an antenna temperature scale. Adopted beam and forward hemisphere efficiencies are 0.80 and 0.95 at $\lambda \sim 3 \mathrm{~mm}$ and 0.62 and 0.92 at $\lambda \sim 1.3 \mathrm{~mm}$. As backend we used fast Fourier transform spectrometers with a channel spacing of $195.3 \mathrm{kHz}$, covering two contiguous $4 \mathrm{GHz}$ segments in dual linear polarization at $\lambda \sim 3$ and also at $\lambda \sim 1.3 \mathrm{~mm}$. Channel spacings were 0.53 and $0.26 \mathrm{~km} \mathrm{~s}^{-1}$, respectively. The spectra were obtained with a wobbling secondary mirror using a switch cycle of a few seconds ( $2 \mathrm{~s}$ on-source, $2 \mathrm{~s}$ off-source) and a beam throw of $\pm 100^{\prime \prime}$. No absorption features are seen in any spectrum, potentially caused by using too small a beam throw. The pointing accuracy, based on nearby continuum sources, was accurate to $\sim 5^{\prime \prime}$. For calibration, see Sect. 3.6.2. Table 1 displays some essential parameters of the observations.

Complementary $\lambda \sim 3$ and $1.3 \mathrm{~mm}$ observations of Mrk 231 were taken in January and May 2011 (project 233-11) with the IRAM 30-m telescope using the same receivers and observing mode with the WILMA backend under varying weather conditions. This backend provided channel spacings of $2 \mathrm{MHz}$.

Data analysis was performed with the GILDAS data reduction package $e^{2}$, revealing excellent baselines only requiring the subtraction of baselines of order $\leq 2$ for both galaxies. The $\lambda=3$ and $1.3 \mathrm{~mm}$ data were taken simultaneously, and the pointing difference between the two EMIR receivers was found to be $\lesssim 2^{\prime \prime}$.

\section{CN and CO toward NGC 253}

\subsection{The galaxy NGC 253}

The Sculptor galaxy NGC 253, an almost edge-on barred spiral ( $i=72^{\circ}-78^{\circ}$; Pence 1981; Puche et al. 1991), is one of the most prolific infrared and molecular lighthouses of the entire extragalactic sky. At a distance of $D \sim 3 \mathrm{Mpc}$ (e.g., Mouhcine et al. 2005; Rekola et al. 2005), it is a prime example of a galaxy with a nuclear starburst devoid of an active galactic nucleus (e.g.,

1 Based on observations carried out with the IRAM 30-m telescope. IRAM is supported by INSU/CNRS (France), the MPG (Germany), and IGN (Spain).

2 Grenoble Image and Line Data Analysis Software: http://www. ira.inaf.it/ brand/gag.html 
Ulvestad \& Antonucci 1997; Henkel et al. 2004). Because of the exceptional strength of its molecular lines, NGC 253 was selected as the target of choice for the first unbiased molecular line survey of an extragalactic source (Martín et al. 2006). It is therefore a highly suitable target for this study.

\subsection{Our data}

$\mathrm{CN}$ spectra are complex. Each $\mathrm{CN}$ rotational energy level with $N>0$ is split into a doublet by spin-rotation interaction. Because of the spin of the nitrogen nucleus $\left(I_{1}=1\right)$, each of these components is then split into a triplet of states. The ${ }^{13} \mathrm{CN}$ spectrum is complicated further by the spin of the ${ }^{13} \mathrm{C}\left(I_{2}=1 / 2\right)$ nucleus.

Figure 1 shows our $\lambda \sim 3 \mathrm{~mm} \mathrm{CN}$ spectra. The lower panel displays the $N=1 \rightarrow 0 J=1 / 2 \rightarrow 1 / 2$ (left) and $J=3 / 2 \rightarrow$ $1 / 2$ (right) groups of lines of ${ }^{12} \mathrm{C}^{14} \mathrm{~N}$ (hereafter $\mathrm{CN}$ ). The upper panel visualizes the blended $N=1 \rightarrow 0 F_{2}=1 \rightarrow 0$ and $1 \rightarrow 1$ (far left) and the slightly weaker $F_{2}=2 \rightarrow 1$ (next feature to the right) transitions of ${ }^{13} \mathrm{C}^{14} \mathrm{~N}$ (hereafter ${ }^{13} \mathrm{CN}$ ). This represents one of the first detections of ${ }^{13} \mathrm{CN}$ in extragalactic space (cf. Aladro et al. 2013; for the first detection in the local interstellar medium, see Gerin et al. 1984). The ${ }^{13} \mathrm{CN}$ spectrum has an rms noise level of $2 \mathrm{mK}$ (channel width: $8.5 \mathrm{~km} \mathrm{~s}^{-1}$ ). While the upper panel of Fig. 1 only shows a small spectral segment, we note that the entire spectrum has a width of $4 \mathrm{GHz}$. Therefore the (flat) baseline and noise level are well defined. Table 2 provides the corresponding line parameters. It should be emphasized that the Gaussian fit result for ${ }^{13} \mathrm{CN}$, fitting the two ${ }^{13} \mathrm{CN}$ profiles and the dominant $\mathrm{CH}_{3} \mathrm{OH}$ feature simultaneously, is very robust.

A comparison with the approximate rest frequencies of the different groups of lines shows that we mainly see the high velocity component of NGC 253 with a recessional velocity of $V_{\mathrm{LSR}} \sim+290 \mathrm{~km} \mathrm{~s}^{-1}$ (e.g., Martín et al. 2006), located several arcseconds southwest of the kinematical center with an extent of order 10" (Mauersberger et al. 1996; Peng et al. 1996; García-Burillo et al. 2000; Paglione et al. 2004; Güsten et al. 2006; Lebrón et al. 2011; Sakamoto et al. 2011; Bolatto et al. 2013). The similarly extended lower velocity component near $170 \mathrm{~km} \mathrm{~s}^{-1}$, mainly arising from several arcseconds northeast of the dynamical center, is too weak to be detected at significant levels (but see Sect. 3.8). For the dominant $0_{0} \rightarrow 1_{-1} E$ line of methanol $\left(\mathrm{CH}_{3} \mathrm{OH}\right)$, we obtain $V_{\mathrm{LSR}}=(294 \pm 4) \mathrm{km} \mathrm{s}^{-1}$. While this inhibits a comparison of the two major molecular lobes near the center of NGC 253, the dominance of the high velocity component in the spectra is nevertheless positive. It reduces the line widths considerably and hence the blending of nearby spectral features.

A search for vibrationally excited $\mathrm{CN}$ turned out to be unsuccessful. The higher frequency fine structure components of the $v=1 N=1 \rightarrow 0$ and $2 \rightarrow 1 \mathrm{CN}$ transitions are blended by ${ }^{12} \mathrm{C}^{17} \mathrm{O} J=1 \rightarrow 0$ and $2 \rightarrow 1$. For the lower frequency fine structure components, we obtain at $\lambda \sim 3$ and $1.3 \mathrm{~mm} 1 \sigma$ noise levels of 3 and $4 \mathrm{mK}$ (15 and $20 \mathrm{mJy}$ ) with a channel width of $8.5 \mathrm{~km} \mathrm{~s}^{-1}$.

\subsection{Importance of $\mathrm{CN}$}

Toward NGC 253 , the ${ }^{12} \mathrm{C} /{ }^{13} \mathrm{C}$ carbon isotope ratio has been previously estimated from CS (Henkel et al. 1993) and $\mathrm{C}_{2} \mathrm{H}$ (Martín et al. 2010). While the former authors propose ${ }^{12} \mathrm{C} /{ }^{13} \mathrm{C} \sim 40$, the latter find ${ }^{12} \mathrm{C} /{ }^{13} \mathrm{C}>81$. In view of the scarcity of ${ }^{12} \mathrm{C} /{ }^{13} \mathrm{C}$ determinations from extragalactic sources, this discrepancy is important to resolve. This is one of the main goals of this paper.
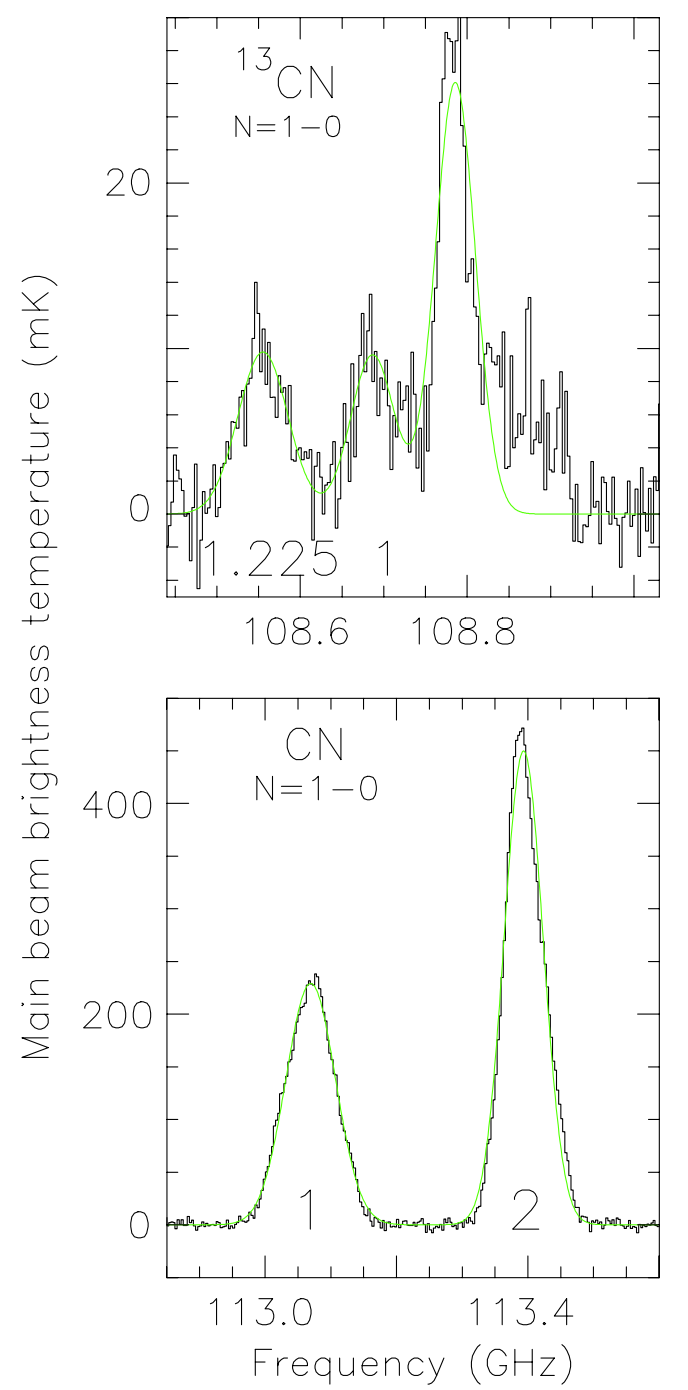

Fig. 1. CN $N=1 \rightarrow 0$ spectra (black) and Gaussian fits (green) from NGC 253 on a local standard of rest (LSR) $V_{\mathrm{LSR}}=0 \mathrm{~km} \mathrm{~s}^{-1}$ frequency scale. Both spectra are smoothed to a channel spacing of $\sim 8.5 \mathrm{~km} \mathrm{~s}^{-1}(3.125 \mathrm{MHz})$. Lower panel: the $J=1 / 2 \rightarrow 1 / 2$ (left) and $J=3 / 2 \rightarrow 1 / 2$ (right) groups of CN lines. Upper panel: the strongest feature is the $0_{0} \rightarrow 1_{-1} \mathrm{E}$ line of $\mathrm{CH}_{3} \mathrm{OH}$ (methanol). Far left: the $F_{1}=0, F_{2}=1 \rightarrow 0$ and $F_{1}=1, F_{2}=1 \rightarrow 1$ groups of ${ }^{13} \mathrm{CN}$ $N=1 \rightarrow 0$ lines. In between this spectral feature and the methanol line: the $F_{1}=1, F_{2}=2 \rightarrow 1$ group of ${ }^{13} \mathrm{CN}$ transitions (for $\mathrm{CN}$ and ${ }^{13} \mathrm{CN}$ rest frequencies, see Skatrud et al. 1983; and Bogey et al. 1984). The emission on the righthand side of the methanol line near $108.9 \mathrm{GHz}$ might be caused by SiS $6 \rightarrow 5$. Numbers at the foot of each spectral CN or ${ }^{13} \mathrm{CN}$ feature provide expected relative intensities with respect to the weaker group of lines in case of optically thin emission under conditions of local thermodynamical equilibrium. For less sensitive $\mathrm{CN}$ spectra obtained with smaller bandwidths, see Fig. 1 of Henkel et al. (1993).

As explained in Sect. 3.2, mm-wave CN spectra contain a multitude of individual features. Therefore, a comparison between the tracer species with highest intensities, $\mathrm{CO}, \mathrm{HCN}$, $\mathrm{HCO}^{+}, \mathrm{HNC}$, and $\mathrm{CN}$, clearly favors $\mathrm{CN}$ when attempting to determine optical depths from relative line intensities to derive carbon isotope ratios (Henkel et al. 1998). This also holds when including $\mathrm{C}_{2} \mathrm{H}$ (Martín et al. 2010), because $\mathrm{CN}$ shows the broadest frequency coverage of spectral fine structure within its mm-wave transitions, which is sufficient even in the case of rotationally broadened lines from an edge-on spiral galaxy (e.g., Fig. 1). 
Table 2. CN line parameters of NGC 253, obtained from Gaussian fits.

\begin{tabular}{ccccc}
\hline \hline Line & $\begin{array}{c}\int T_{\mathrm{mb}} \\
(\mathrm{K} \mathrm{MHz})\end{array}$ & $\begin{array}{c}v \\
(\mathrm{MHz})\end{array}$ & $\begin{array}{c}\Delta v_{1 / 2} \\
(\mathrm{MHz})\end{array}$ & $\begin{array}{c}T_{\mathrm{mb}} \\
(\mathrm{mK})\end{array}$ \\
\hline $\mathrm{CN} N=1 \rightarrow 0$ & $21.637 \pm 0.083$ & $113069.244 \pm 0.166$ & $88.721 \pm 0.388$ & $244 \pm 1$ \\
& $33.537 \pm 0.061$ & $113393.540 \pm 0.063$ & $70.041 \pm 0.170$ & $479 \pm 1$ \\
& & & & \\
${ }^{13} \mathrm{CN}=1 \rightarrow 0$ & $0.736 \pm 0.086$ & $108555.278 \pm 3.931$ & $70.565 \pm 9.396$ & $10.4 \pm 1.8$ \\
& $0.632 \pm 0.091$ & $108686.718 \pm 4.555$ & $61.822 \pm 10.458$ & $10.2 \pm 2.3$ \\
$\mathrm{CH}_{3} \mathrm{OH} 0_{0} \rightarrow 1_{-1} \mathrm{E}$ & $1.569 \pm 0.108$ & $108785.756 \pm 1.333$ & $56.561 \pm 6.061$ & $27.7 \pm 3.5$ \\
& & & & \\
$\mathrm{CN} N=2 \rightarrow 1$ & $15.009 \pm 0.160$ & $226138.445 \pm 0.556$ & $116.099 \pm 1.369$ & $129 \pm 2$ \\
& $29.210 \pm 0.167$ & $226518.547 \pm 0.324$ & $115.337 \pm 0.750$ & $253 \pm 2$ \\
& $47.099 \pm 0.164$ & $226676.359 \pm 0.174$ & $105.801 \pm 0.463$ & $445 \pm 2$ \\
\hline
\end{tabular}

Notes. Because of large bandwidths (4-8 GHz) that yield potentially complex velocity-frequency correlations, all fits were obtained on a frequency scale. All fitted $\mathrm{CN}$ components refer to groups of individual lines. Column 3 displays observed frequencies, referring to the local standard of rest (LSR) $V_{\mathrm{LSR}}=0 \mathrm{~km} \mathrm{~s}^{-1}$ frequency scale. All given errors are standard deviations obtained from Gaussian fits. The $T_{\mathrm{mb}}$ values (last column) were obtained from the values given in Cols. 2 and 4. Calibration uncertainties are not considered here but are discussed in Sect. 3.6.2.

\subsection{Problems related to previous ${ }^{12} \mathrm{C} /{ }^{3} \mathrm{C}$ determinations}

The ${ }^{12} \mathrm{C} /{ }^{13} \mathrm{C} \sim 40$ estimate from CS by Henkel et al. (1993) for NGC 253 was based on the assumption of ${ }^{32} \mathrm{~S} /{ }^{34} \mathrm{~S}$ ratio of 23 as measured in the solar system and the local interstellar medium (Penzias 1980; Wannier 1980). More recent data, however, indicate a strong positive ${ }^{32} \mathrm{~S} /{ }^{34} \mathrm{~S}$ gradient in the Galactic disk (Chin et al. 1996) with ${ }^{32} \mathrm{~S} /{ }^{34} \mathrm{~S}$ of order 13.5 in the inner disk and a possibly similar ratio in the nuclear starburst environment of another active nearby spiral galaxy, NGC 4945 (Wang et al. 2004).

For a ratio of ${ }^{32} \mathrm{~S} /{ }^{34} \mathrm{~S}=13.5$, following the procedure outlined by Henkel et al. (1993), we would obtain ${ }^{12} \mathrm{C} /{ }^{13} \mathrm{C} \sim 23$ for NGC 253. More recently, Martín et al. (2005, 2006) obtained $I\left({ }^{12} \mathrm{C}^{32} \mathrm{~S} 3-2\right) / I\left({ }^{12} \mathrm{C}^{34} \mathrm{~S} 3-2\right) \sim 5.7$ and $I\left({ }^{12} \mathrm{C}^{32} \mathrm{~S} 3-2\right) / I\left({ }^{13} \mathrm{C}^{32} \mathrm{~S}\right.$ $3-2) \sim 27$. (The ratios were calculated from their Tables 1, giving integrated intensities, while Martín et al. 2005, discuss $I\left({ }^{12} C^{32} S 3-2\right) / I\left({ }^{13} C^{32} S 3-2\right)=21 \pm 3$ in their Sect. 3.1, derived from peak flux densities.) With the data from their Table 1 and ${ }^{32} \mathrm{~S} /{ }^{34} \mathrm{~S}=13.5$, we obtain, following the same procedure (Henkel et al. 1993), from CS a carbon isotope ratio of ${ }^{12} \mathrm{C} /{ }^{13} \mathrm{C} \sim 70$ for NGC 253.

In this context it has to be mentioned that ${ }^{32} \mathrm{~S} /{ }^{34} \mathrm{~S}=8 \pm 2$, as suggested by Martín et al. (2005), cannot be adopted because it has been derived from the ${ }^{12} \mathrm{C} /{ }^{13} \mathrm{C} \sim 40$ ratio proposed by Henkel et al. (1993) under the assumption of ${ }^{32} \mathrm{~S} /{ }^{34} \mathrm{~S}=23$. However, the sulfur isotope ratio of $>16$, suggested more recently by Martín et al. (2010), is free of such a contradiction. To summarize, the poorly constrained sulfur isotope ratio in NGC 253 and the (initially unknown) strong Galactic ${ }^{32} \mathrm{~S} /{ }^{34} \mathrm{~S}$ gradient (Chin et al. 1996), allowing for a wide range of ratios at least in the Galaxy, inhibit any reliable determination of NGC 253's carbon isotope ratio based on CS. In view of the qualitative nature of the abovementioned ratios, a systematic analysis of error budgets is not feasible.

\subsection{Estimating ${ }^{12} \mathrm{CN} /{ }^{13} \mathrm{CN}$, excitation temperature, and column density}

With two detected groups of features in the $\mathrm{CN}$ and ${ }^{13} \mathrm{CN} N=$ $1 \rightarrow 0$ lines (Fig. 1) and three in the $\mathrm{CN} N=2 \rightarrow 1$ transition (Fig. 2), we are able to quantify opacity effects without having to rely on a second isotope ratio (like that of ${ }^{32} \mathrm{~S} /{ }^{34} \mathrm{~S}$ ). Therefore, $\mathrm{CN}$ provides a good data base to directly estimate the ${ }^{12} \mathrm{C} /{ }^{13} \mathrm{C}$ ratio in NGC 253. If local thermodynamical equilibrium (LTE)

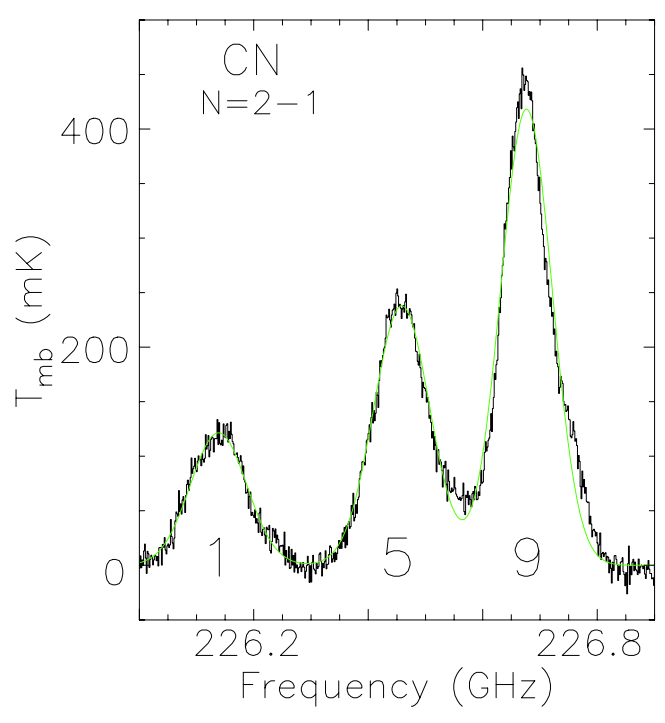

Fig. 2. CN $N=2 \rightarrow 1$ spectrum (black) and Gaussian fits (green) of NGC 253 in units of main beam brightness temperature on a LSR $V_{\mathrm{LSR}}=0 \mathrm{~km} \mathrm{~s}^{-1}$ frequency scale. The profile has been smoothed to a channel spacing of $\sim 2.1 \mathrm{~km} \mathrm{~s}^{-1}(1.5625 \mathrm{MHz})$. Numbers at the foot of each spectral feature provide expected relative intensities with respect to the weakest group of lines in case of optically thin emission under conditions of local thermodynamical equilibrium.

holds and lines are optically thin, line intensity ratios should be $1: 2(\mathrm{CN} N=1 \rightarrow 0), 1.225: 1\left({ }^{13} \mathrm{CN} N=1 \rightarrow 0\right)$, and 1:5:9 $(\mathrm{CN}$ $N=2 \rightarrow 1$ ), when moving from left to right with increasing frequency in Figs. 1 and 2.

Dividing the integrated intensities of the $\mathrm{CN}$ and ${ }^{13} \mathrm{CN} N=$ $1 \rightarrow 0$ transitions, we obtain $I(\mathrm{CN}) / I\left({ }^{13} \mathrm{CN}\right)=40$. Also accounting for the $F_{1}, F_{2}=0 \rightarrow 1{ }^{13} \mathrm{CN}$ components near 108.4 , which are not seen but contribute $7.6 \%$ to the total ${ }^{13} \mathrm{CN}$ emission in the case of optically thin lines and prevailing LTE conditions (see Bogey et al. 1984), the ratio drops to $I(\mathrm{CN}) / I\left({ }^{13} \mathrm{CN}\right)=37.5$ for the $N=1 \rightarrow 0$ transition.

To estimate the $\mathrm{CN}$ excitation temperature, we note that the $N=2 \rightarrow 1 / 1 \rightarrow 0$ line intensity ratio is 1.655 (Table 2 ) on a frequency scale and 0.83 on a velocity scale. The latter is relevant here. Accounting for the fact that the $\mathrm{CN} N=2 \rightarrow 1$ linear beam size $\theta_{\mathrm{b}}$ is half that of the $N=1 \rightarrow 0$ line and assuming that the source size is small with respect to $\theta_{\mathrm{b}, \mathrm{CN} 2-1}$, beam 
dilution is four times higher for the $N=1 \rightarrow 0$ than for the $2 \rightarrow 1$ transition. The corrected line intensity ratio then becomes 0.21 . Figure 2c of Mauersberger et al. (1996) suggests that the high velocity $\mathrm{CO} J=2 \rightarrow 1$ emission has an extent comparable to $\theta_{\mathrm{b}, \mathrm{CN} 2-1}$. Since $\mathrm{CN}$ may arise from an even more compact region, this provides an upper limit to the possible extent of the $\mathrm{CN}$ emission. Therefore the real CN $N=2 \rightarrow 1 / 1 \rightarrow 0$ intensity ratio may be close to 0.25 , but below we account for the entire range of possible values. For optically thin emission, we use the $N=2 \rightarrow / 1 \rightarrow 0$ ratio to constrain the excitation temperature via

$0.25=4 \mathrm{e}^{-\mathrm{x}} \times \frac{1-\mathrm{e}^{-2 \mathrm{x}}}{1-\mathrm{e}^{-\mathrm{x}}} \times \frac{\left(\mathrm{e}^{2 \mathrm{x}}-1\right)^{-1}-\left(\mathrm{e}^{2 \mathrm{y}}-1\right)^{-1}}{\left(\mathrm{e}^{\mathrm{x}}-1\right)^{-1}-\left(\mathrm{e}^{\mathrm{y}}-1\right)^{-1}}$

(e.g., Wang et al. 2004), where $x=h v_{10} / k T_{\mathrm{ex}}, v_{10}=$ $113.386 \mathrm{GHz}$ (an averaged $\mathrm{CN} N=1 \rightarrow 0$ rest frequency), and $y=h v_{10} / 2.73 k=1.99$. In this way we derive an excitation temperature of $T_{\mathrm{ex}}=3.5_{-0.3}^{+3.0} \mathrm{~K}$. The error limits account for the entire range of possible line intensity ratios from 0.21 to 0.83 . For an estimate also discussing effects of line saturation and non-LTE excitation, see Sect. 3.7.

\subsection{Potential uncertainties in the derived carbon isotope ratio}

Toward NGC $253,{ }^{12} \mathrm{C} /{ }^{13} \mathrm{C}$ line intensity ratios from carbonbearing species with well detected ${ }^{13} \mathrm{C}$-containing isotopologues are given by Henkel et al. (1993) for CO $J=1 \rightarrow 0$ and $2 \rightarrow 1$, $\mathrm{HCN} J=1 \rightarrow 0, \mathrm{HCO}^{+} J=1 \rightarrow 0$, and CS $J=3 \rightarrow 2$. These ratios do not surpass 20 and can be taken as lower limits in view of the unknown but certainly substantial optical depths of the main isotopologues. Martín et al. $(2005,2006)$ find (as already mentioned) a ratio of 27 for $\mathrm{CS} J=3 \rightarrow 2$. In view of all these data, our determination of the $\mathrm{CN} /{ }^{13} \mathrm{CN} N=1 \rightarrow 0$ line intensity ratio of order 40 is a major step ahead. However, questions related to fractionation, isotope selective photodissociation, calibration, and optical depths of the main $\mathrm{CN}$ species still remain to be discussed.

\subsubsection{Chemical fractionation and isotope selective photodissociation}

Langer et al. (1984) modeled the fractionation of oxygen and carbon in dense interstellar clouds with time-dependent chemistry, involving cloud lifetimes up to $10^{8} \mathrm{yr}$, kinetic temperatures of $6-80 \mathrm{~K}$, and densities of $5 \times 10^{2}-10^{5} \mathrm{~cm}^{-3}$ for a wide range of metal abundances. While oxygen isotope fractionation is insignificant under all considered conditions, carbon fractionation occurs, resulting in $\mathrm{CO}$ providing too low ${ }^{12} \mathrm{C} /{ }^{13} \mathrm{C}$ ratios, $\mathrm{HCO}^{+}$ delivering quite accurate ones, and $\mathrm{CN}, \mathrm{CS}, \mathrm{HCN}$, and $\mathrm{H}_{2} \mathrm{CO}$ yielding them too high.

Observations of $\mathrm{H}_{2} \mathrm{CO}, \mathrm{C}^{18} \mathrm{O}$, and $\mathrm{CN}$, including the corresponding ${ }^{13} \mathrm{C}$-bearing species, across the Galactic plane (Henkel et al. 1982; Langer \& Penzias 1990; Milam et al. 2005) demonstrate how large the numerically predicted discrepancies may become in the real world. Observationally, the carbon isotope ratios from $\mathrm{H}_{2} \mathrm{CO}$ turn out to be higher by $\sim 30 \%$ than those from $\mathrm{C}^{18} \mathrm{O}$ and $\mathrm{CN}$, which are quite similar. Finding a synthesis for all these data sets, Milam et al. (2005) conclude that there is good agreement between all measured ${ }^{12} \mathrm{C} /{ }^{13} \mathrm{C}$ values at a given galactocentric radius, independent of the kinetic temperature of the cloud observed. Thus chemical fractionation and isotope selective photodissociation do not play a dominant role.
In addition to the advantage of $\mathrm{CN}$ showing a peculiar spectral fine structure (see Sect. 3.3), we thus also find that there is an exemplary Galactic data set on $\mathrm{CN} /{ }^{13} \mathrm{CN}$ (Milam et al. 2005). There is no such analog for $\mathrm{C}_{2} \mathrm{H}$, encompassing the Galactic disk and center, which would be relevant for the analysis of NGC 253 by Martín et al. (2010). Since $\mathrm{C}_{2} \mathrm{H}$ is a molecule, which may also permit deriving line opacities of the main isotopologue in galaxies with moderate line widths, a systematic survey of $\mathrm{C}_{2} \mathrm{H}$, ${ }^{13} \mathrm{CCH}$, and $\mathrm{C}^{13} \mathrm{CH}$ across the Galaxy would be highly desirable by providing interesting insights into astrochemistry, possibly delivering a benchmark for extragalactic data, and allowing us to critically compare carbon ratios derived from $\mathrm{CN}$ and $\mathrm{C}_{2} \mathrm{H}$ also in extragalactic sources. In view of similar results from $\mathrm{H}_{2} \mathrm{CO}, \mathrm{C}^{18} \mathrm{O}$, and $\mathrm{CN}$ in the Galaxy (Milam et al. 2005), we expect compatible results from $\mathrm{C}_{2} \mathrm{H}$ as well, but this has still to be demonstrated in a rigorous way. Although it would be unexpected (see, e.g., Wang et al. 2009, who find that the LMC is well mixed with respect to the carbon isotope ratio), we can also not yet firmly exclude that $\mathrm{CN}$ and $\mathrm{C}_{2} \mathrm{H}$ trace different regions with different ${ }^{12} \mathrm{C} /{ }^{13} \mathrm{C}$ values.

Deviations from relative LTE intensities of different features within the $N=1 \rightarrow 0$ or $2 \rightarrow 1$ transitions of $\mathrm{C}_{2} \mathrm{H}$ have been found to be small (e.g., Padovani et al. 2009), suggesting that $\mathrm{C}_{2} \mathrm{H}$ will be useful for studies of isotope ratios. Furthermore, $\mathrm{CN}$ and $\mathrm{C}_{2} \mathrm{H}$ appear to be chemically related. Both are common tracers of dark clouds (e.g., Padovani et al. 2009, and references therein) and are also probes of photon-dominated regions (PDRs; e.g., Simon et al. 1997; Rimmer et al. 2012). Because of high critical densities (for $\mathrm{C}_{2} \mathrm{H}$, see Spielfiedel et al. 2012; for $\mathrm{CN}$, see Sect. 3.7), their molecular line emission should be weak in clouds of low density. In view of all these common properties, significantly different ${ }^{12} \mathrm{C} /{ }^{13} \mathrm{C}$ ratios from $\mathrm{CN}$ and $\mathrm{C}_{2} \mathrm{H}$ would be a great surprise.

\subsubsection{Calibration}

Table 1 shows a drastic difference between the system temperatures of the two $\lambda \sim 3 \mathrm{~mm}$ spectra centered at 109.635 and $113.365 \mathrm{GHz}$. This is mainly a consequence of the extinction caused by atmospheric $\mathrm{O}_{2}$ near $118 \mathrm{GHz}$. While previous studies were made with comparatively small bandwidths, here we face the problem that a single system temperature stands for a spectrum with supposedly quite different atmospheric extinctions at its low and high frequency edges. Because of this, we have to compare our measured main beam brightness temperatures with those of previous studies.

Only data from the 30-m IRAM telescope are considered here. With respect to ${ }^{13} \mathrm{C}^{16} \mathrm{O}$ (hereafter ${ }^{13} \mathrm{CO}$ ) and ${ }^{12} \mathrm{C}^{18} \mathrm{O}$ (hereafter $\mathrm{C}^{18} \mathrm{O}$ ) $J=1 \rightarrow 0$ near $110 \mathrm{GHz}$ (Fig. 3), agreement with the profiles of Harrison et al. (1999) is excellent. When compared with Fig. 1 of Henkel et al. (1993), this also holds for the two main groups of features of the $\mathrm{CN} N=2 \rightarrow 1$ transition near $226 \mathrm{GHz}$. While our signal-to-noise ratios are much higher, there is also no notable discrepancy between Fig. 1 of Henkel et al. (1993) and our ${ }^{13} \mathrm{CN}$ spectrum near $108.6 \mathrm{GHz}$ (Fig. 1, upper panel). This mainly refers to the $\mathrm{CH}_{3} \mathrm{OH} \mathrm{O}_{0} \rightarrow 1_{-1}$ line, because the noise level in the previously published spectrum is too high to detect ${ }^{13} \mathrm{CN}$. Here we should note that all lines considered so far are displaced well away from the atmospheric $118 \mathrm{GHz}$ $\mathrm{O}_{2}$ feature so that atmospheric extinction is not expected to vary strongly within the individually observed $4 \mathrm{GHz}$ wide frequency bands.

Nevertheless, there are also inconsistencies. Henkel et al. (1988) measured only $T_{\mathrm{mb}} \sim 250 \mathrm{mK}$ for $\mathrm{CN} N=2 \rightarrow 1$, which 


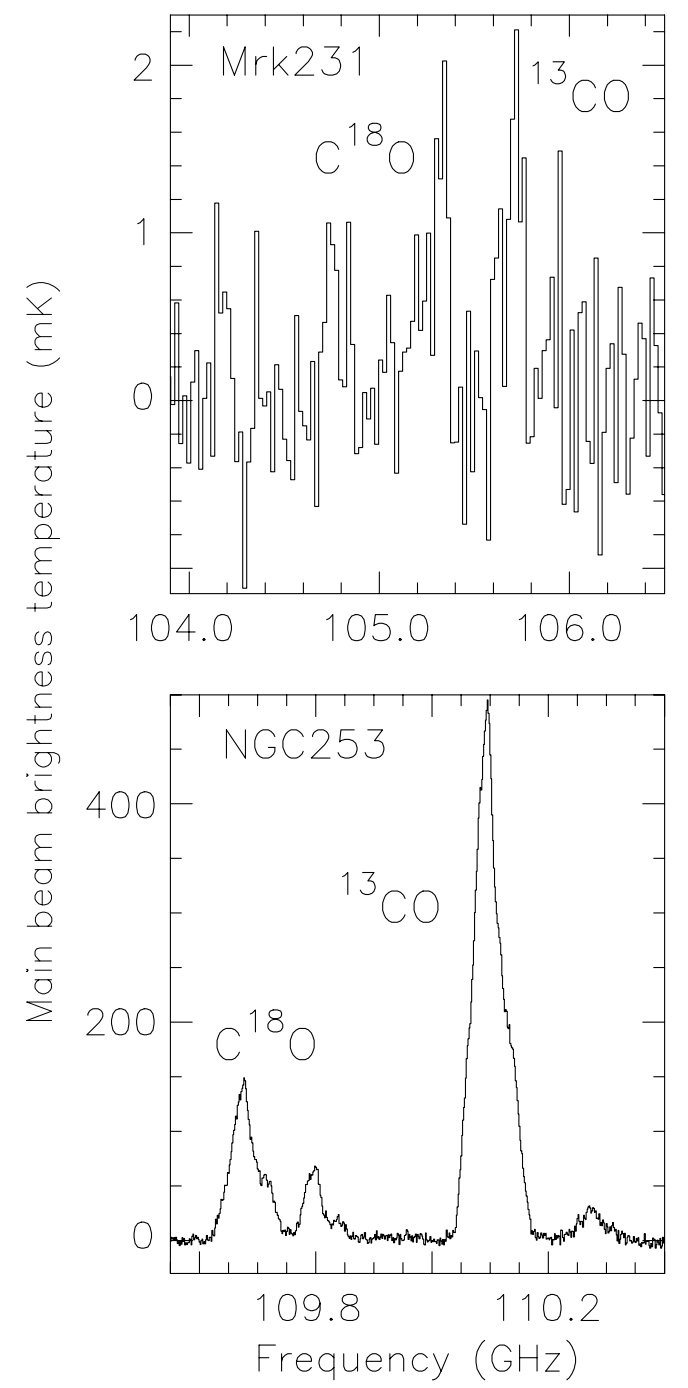

Fig. 3. $\mathrm{C}^{18} \mathrm{O}$ and ${ }^{13} \mathrm{CO} J=1 \rightarrow 0$ spectra from NGC 253 (lower panel) and Mrk 231 (upper panel) on a LSR $V_{\mathrm{LSR}}=0 \mathrm{~km} \mathrm{~s}^{-1}$ frequency scale. The spectra were smoothed to channel spacings of $\sim 4.26$ and $\sim 60.0 \mathrm{~km} \mathrm{~s}^{-1}$ (1.53 and $21.0 \mathrm{MHz}$ ), respectively. In the lower panel, the features at 109.8 and $110.27 \mathrm{GHz}$ belong to HNCO $J=5 \rightarrow 4$ and $\mathrm{CH}_{3} \mathrm{CN} J=6 \rightarrow 5$.

is lower than what Henkel et al. (1993) reported five years later and what we have observed in this study ( $450 \mathrm{mK}$; cf. Table 2$)$. The IRAM 30-m telescope has been greatly improved during the years between 1988 and 1993, so the later observation should be preferred, providing support for our spectrum displayed in Fig. 2. More critical is $\mathrm{CN} N=1 \rightarrow 0$, because it has been observed in the $4 \mathrm{GHz}$ wide band also covering $\mathrm{CO} J=1 \rightarrow 0$ at the edge of the atmospheric $118 \mathrm{GHz} \mathrm{O}$ feature. Henkel et al. (1988, 1993) find $T_{\mathrm{mb}} \sim 300$ and $350 \mathrm{mK}$ for CN $N=1 \rightarrow 0$, which is $\sim 40 \%$ and $25 \%$ below our value (Table 2 ). With the $\mathrm{CN} \mathrm{N}=1 \rightarrow 0$ frequencies being located in between those of ${ }^{13} \mathrm{CO}$ and $\mathrm{C}^{18} \mathrm{O} J=1 \rightarrow 0$ and $\mathrm{CO} J=1 \rightarrow 0$, our $\mathrm{CO}$ peak intensity is also relevant for an evaluation of calibration uncertainties. With $T_{\mathrm{mb}} \sim 5.7$ (Fig. 4), we obtain a value well above the one shown in Fig. 5c of Mauersberger et al. (1996), reaching only $\sim 4 \mathrm{~K}$ for the $V_{\mathrm{LSR}} \sim+290 \mathrm{~km} \mathrm{~s}^{-1}$ velocity component seen by us (Sect. 3.2). This difference of $\sim 30 \%$ relative to our result is similar to the deviation obtained for $\mathrm{CN} N=1 \rightarrow 0$ and will be implemented as the main uncertainty in our estimate of the carbon isotope ratio (see Sect. 3.7).
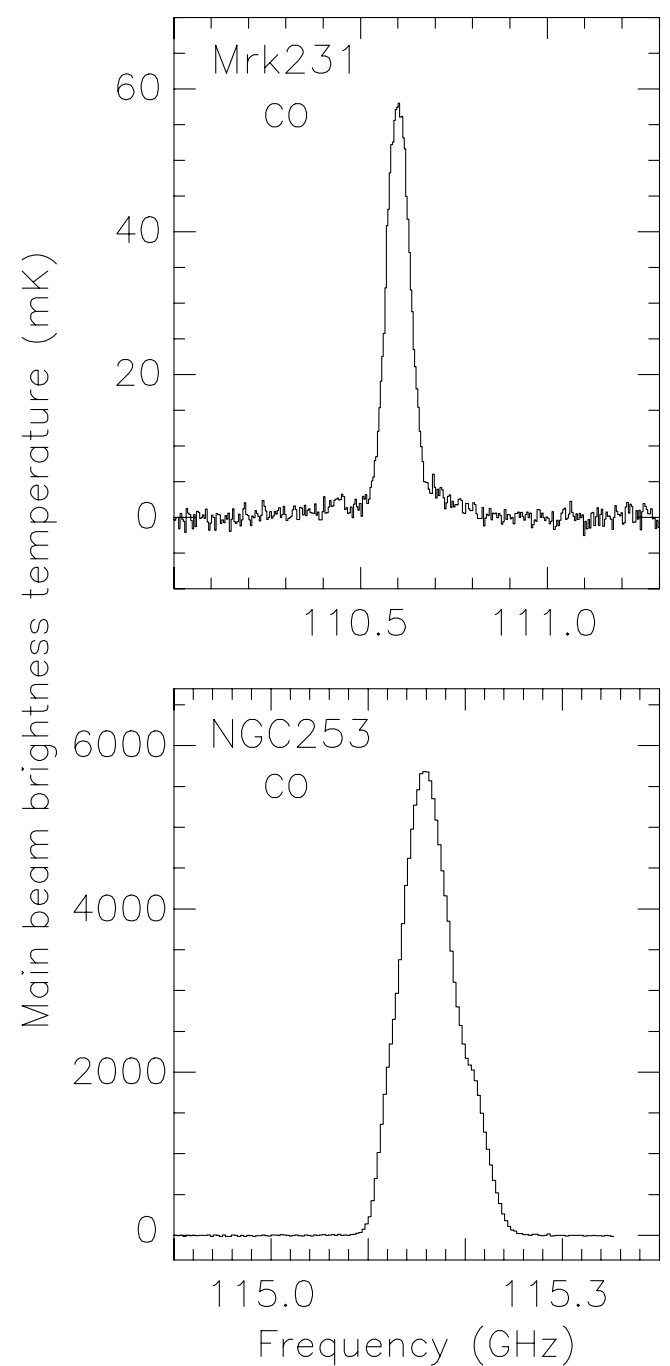

Fig. 4. CO $J=1 \rightarrow 0$ spectra from NGC 253 (lower panel) and Mrk 231 (upper panel) on a LSR $V_{\mathrm{LSR}}=0 \mathrm{~km} \mathrm{~s}^{-1}$ frequency scale. The spectra were smoothed to channel spacings of $\sim 8.52$ and $10.84 \mathrm{~km} \mathrm{~s}^{-1}$ ( 3.125 and $4.0 \mathrm{MHz})$, respectively. The frequency range of the upper panel is wider to show the molecular outflow at the foot of the main spectral component, seen in $\mathrm{CO}$ and other species (e.g., Feruglio et al. 2010; Aalto et al. 2012).

\subsubsection{Optical depths}

$\mathrm{CN}$ has been observed in several clouds of the Galactic center region (Henkel et al. 1998), which is as close as we can get to the physical conditions prevailing in the nuclear region of NGC 253. Toward cloud cores, deviations of the individual spectral features from LTE were found to be moderate. Integrated intensity ratios are $I(\mathrm{CN}) / I\left({ }^{13} \mathrm{CN}\right)=9-15$ in the $N=1 \rightarrow 0$ line, which is below the canonical Galactic center ratio of ${ }^{12} \mathrm{C} /{ }^{13} \mathrm{C} \sim 25$, indicating moderate $\mathrm{CN}$ saturation. In this context, we should also mention the interferometric $\mathrm{CN}$ observations of the innermost $4 \mathrm{pc}$ of our Galaxy (Martín et al. 2012), which yield carbon isotope ratios of $15-45$. We note, however, that this study addresses a very small region compared to those discussed here.

In NGC 253, the line intensity ratio of the two ${ }^{13} \mathrm{CN} N=$ $1 \rightarrow 0$ features is $1.165 \pm 0.215$, which is consistent with LTE and optically thin emission (Fig. 1, upper panel). We may, however, face some moderate saturation in the $\mathrm{CN} N=1 \rightarrow 0$ line. Instead of the 2-to-1 ratio expected under LTE conditions with optically thin lines, the ratio of integrated intensities between 


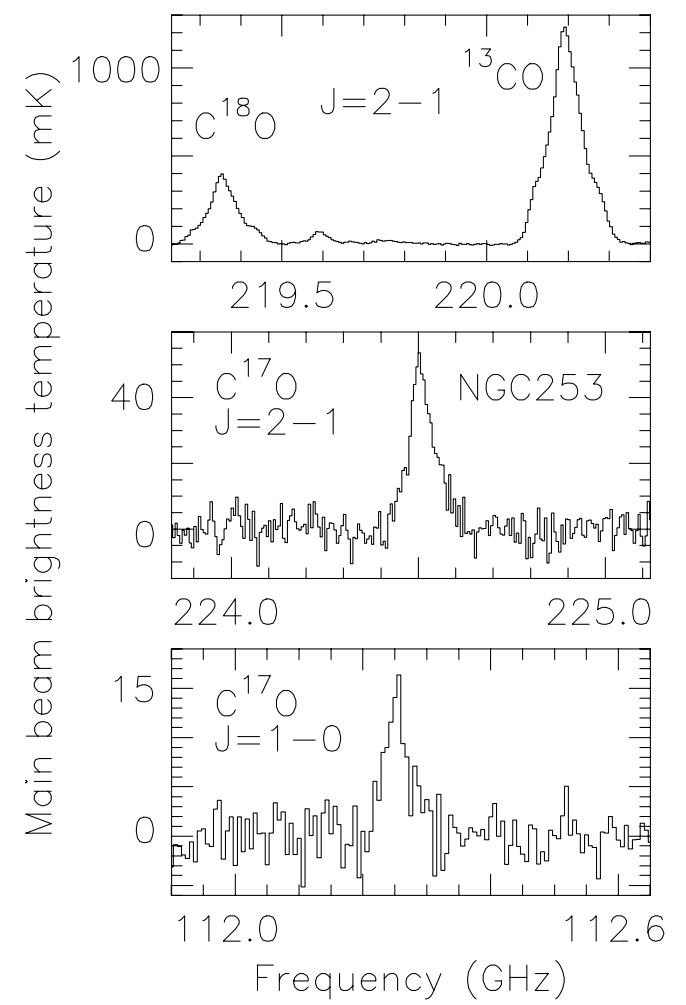

Fig. 5. ${ }^{13} \mathrm{CO}, \mathrm{C}^{18} \mathrm{O}$, and $\mathrm{C}^{17} \mathrm{O}$ spectra from NGC 253 (see also Figs. 3 and 4) on a LSR $V_{\mathrm{LSR}}=0 \mathrm{~km} \mathrm{~s}^{-1}$ frequency scale. The profiles were smoothed to channel spacings of $\sim 8.5,8.5$, and $17 \mathrm{~km} \mathrm{~s}^{-1}$ from top to bottom, which corresponds to $6.25 \mathrm{MHz}$.

the $J=3 / 2 \rightarrow 1 / 2$ and $1 / 2 \rightarrow 1 / 2$ groups of lines is $1.55 \pm 0.01$ (see Fig. 1 and Table 2). We note, however, that the peak line intensity ratio between the two groups of line components is almost exactly two. The stronger spectral component is narrower, a consequence of the relative frequencies of its individual hyperfine components. To get twice the integrated intensity, the stronger component would have to have a peak temperature of $T_{\mathrm{mb}}=606 \mathrm{mK}, \sim 2.5$ times the $T_{\mathrm{mb}}$ value of the weaker component. With the observed $479 \mathrm{mK}$ instead (Table 2) and assuming equal excitation temperatures for all individual features, we can apply the radiative transfer equation to determine the optical depth via

$606 / 479 \sim 1.265=\frac{\tau}{\left(1-\mathrm{e}^{-\tau}\right)}$.

This gives a peak optical depth of $\tau \sim 0.5$. For the other measured CN $1 \rightarrow 0$ feature, we then obtain an optical depth of $\tau \sim 0.2$, and effects due to saturation should amount to $\sim 26.5 \%$ and $10 \%$, respectively.

\subsection{Consequences}

To determine the carbon isotope ratio, we thus multiply the integrated intensity $I_{113.1}$ of the weaker $\mathrm{CN} N=1 \rightarrow 0$ feature by $f_{113.1}=1.1$ and that of the stronger one, $I_{113.4}$, by $f_{113.4}=1.265$ to account for line saturation (here and below, the indices refer to redshifted frequencies in units of $\mathrm{GHz}$ ). With the values given in Table 2 and the correction to the measured $I_{13 \mathrm{CN}}$ intensity mentioned in Sect. 3.5, we then obtain a ${ }^{12} \mathrm{CN} /{ }^{13} \mathrm{CN} N=1 \rightarrow 0$ ratio of

$\frac{\left(I_{113.1} \times f_{113.1}\right)+\left(I_{113.4} \times f_{113.4}\right)}{1.082 \times I_{13 \mathrm{CN}}}=45$.
This should be consistent with the carbon isotope ratio (e.g., Milam et al. 2005). The main error could be caused by an overestimate of our $\mathrm{CN} 1 \rightarrow 0$ main beam brightness temperature scale by $\sim 30 \%$, as outlined in Sect. 3.6.2. This would reduce the isotope ratio to a value of approximately 30 . Since all other errors appear negligible relative to this one, we conclude that the carbon isotope ratio is 30-50 in the southwestern starburst core of NGC 253. This is well below the lower limit proposed by Martín et al. (2010), while it is perfectly consistent with the ratio derived by Henkel et al. (1993) from CS. Nevertheless, we consider this latter agreement as fortuitous. It implies that the ${ }^{32} \mathrm{~S} /{ }^{34} \mathrm{~S}$ sulfur isotope ratio in the central part of NGC 253 is close to the local interstellar value, in agreement with $>16$, the value suggested by Martín et al. (2010). While all this is highly consistent and straightforward, we still have to emphasize that the derived carbon isotope ratio is based on the assumption that the intrinsic $\mathrm{CN} N=1 \rightarrow 0$ relative line strengths are, like those of ${ }^{13} \mathrm{CN} 1 \rightarrow 0$, close to their LTE values (for further support for this assumption, see Sect. 4.1).

With $f_{113.1}$ and $f_{113.4}$, accounting for saturation effects in the $\mathrm{CN} 1 \rightarrow 0$ transition, and neglecting those eventually existing in the $N=2 \rightarrow 1$ line, we can now also re-evaluate the $\mathrm{CN}$ excitation temperature from a modified $\mathrm{CN} N=2 \rightarrow 1 / 1 \rightarrow 0$ intensity ratio, which drops from 0.83 to 0.69 on a velocity scale (see Sect. 3.5). This line ratio would be reduced to 0.172 in case of a point source due to the different beam-filling factors of the two lines. Following the discussion in Sect. 3.5 and adopting a ratio of 0.2 , this results in $T_{\mathrm{ex}}=3.2_{-0.2}^{+2.4} \mathrm{~K}$ with the given errors covering the entire range of allowed line ratios from 0.172 to 0.69 .

The intensity ratio of the two main $\mathrm{CN} N=2 \rightarrow 1 \mathrm{spec}-$ tral features (Fig. 2) is close to the LTE value of 1.8:1. Their ratio of peak intensities is $1.76 \pm 0.02$ and the ratio of integrated intensities is $1.61 \pm 0.01$, while line widths are similar. This suggests that saturation only plays a minor role, affecting line intensities by $\lesssim 10 \%$, as is also suggested by the moderate opacities of the $1 \rightarrow 0$ lines and the low excitation temperature derived above. This effectively reduces populations in the higher $N$-levels. While all this is perfectly consistent, the intensity of the weakest $2 \rightarrow 1$ feature (Fig. 2) diverges significantly. This component appears to be far too strong relative to the others.

An alternative view of the $N=2 \rightarrow 1$ line (see Fig. 2) would be that the strongest and weakest $N=2 \rightarrow 1$ features are in LTE, while the central feature is depleted by a non-LTE effect. Then saturation effects would reduce the main beam brightness temperature of the strongest feature from $1274 \mathrm{mK}$ (nine times the peak intensity of the weakest feature multiplied by the ratio of the two line widths, 1.097; see Fig. 2 and Table 2) to the observed $445 \mathrm{mK}$, implying a peak optical depth of $\tau \sim 3.7$. Calculating the excitation temperature in this way by adopting half this peak optical depth for the weaker central feature, we obtain, with the values of Table 2, i.e. integrating over frequency, a $N=2 \rightarrow 1 / 1 \rightarrow 0$ line intensity ratio of

$$
\frac{\left(I_{226.7} \times f_{226.7}\right)+\left(I_{226.5} \times f_{226.5}\right)+I_{226.1}}{\left(I_{113.1} \times f_{113.1}\right)+\left(I_{113.4} \times f_{113.4}\right)}=3.23 \text {. }
$$

For the quantities in the denominator, see the previous equation. For the numerator, we have $f_{226.7}=2.86$ and $f_{226.5}=2.20$. Integrating not over frequency but over velocity, which is the proper unit, yields half the $\mathrm{CN}$ line intensity ratio, 1.614, or, for a point source (see Sect. 3.5), 0.403. The resulting excitation then becomes $4.2 \mathrm{~K}<T_{\mathrm{ex}}<11.3 \mathrm{~K}$. Because the $\mathrm{CN}$ emission is probably not extended with respect to the beam (Sect. 3.5), the actual excitation temperature should be close to $4 \mathrm{~K}$, still a very 
A\&A 565, A3 (2014)

Table 3. CO line parameters for NGC 253 and CO and CN line parameters for Mrk 231, obtained from Gaussian fits.

\begin{tabular}{|c|c|c|c|c|c|}
\hline Line & $\int_{(\mathrm{K} \mathrm{MHz})} T_{\mathrm{mb}}$ & $\begin{array}{c}V \\
\left(\mathrm{~km} \mathrm{~s}^{-1}\right)\end{array}$ & $\begin{array}{c}v \\
(\mathrm{MHz})\end{array}$ & $\begin{array}{r}\Delta v_{1 / 2} \\
(\mathrm{MHz})\end{array}$ & $\begin{array}{r}T_{\mathrm{mb}} \\
(\mathrm{mK})\end{array}$ \\
\hline \multicolumn{6}{|l|}{$N G C 253$} \\
\hline${ }^{12} \mathrm{C}^{16} \mathrm{O} J=1 \rightarrow 0$ & $403.040 \pm 0.125$ & $285 \pm 1$ & $115161.571 \pm 0.010$ & $68.931 \pm 0.025$ & $5847 \pm 3$ \\
\hline Comp. 1 & $360.970 \pm 0.149$ & $293 \pm 1$ & $115158.298 \pm 0.002$ & $59.460 \pm 0.029$ & $6070 \pm 4$ \\
\hline Comp. 2 & $45.321 \pm 0.121$ & $155 \pm 1$ & $115211.774 \pm 0.032$ & $35.844 \pm 0.092$ & $1264 \pm 5$ \\
\hline${ }^{13} \mathrm{C}^{16} \mathrm{O} J=1 \rightarrow 0$ & $28.840 \pm 0.070$ & $285 \pm 1$ & $110096.675 \pm 0.075$ & $63.038 \pm 0.184$ & $458 \pm 2$ \\
\hline Comp. 1 & $24.349 \pm 0.075$ & $300 \pm 1$ & $110092.448 \pm 0.072$ & $49.949 \pm 0.172$ & $487 \pm 2$ \\
\hline Comp. 2 & $4.593 \pm 0.066$ & $167 \pm 1$ & $110139.946 \pm 0.179$ & $32.367 \pm 0.358$ & $142 \pm 3$ \\
\hline${ }^{12} \mathrm{C}^{18} \mathrm{O} J=1 \rightarrow 0$ & $8.009 \pm 0.101$ & $285 \pm 1$ & $109677.878 \pm 0.372$ & $60.921 \pm 0.949$ & $131 \pm 3$ \\
\hline Comp. 1 & $6.652 \pm 0.084$ & $297 \pm 1$ & $109673.695 \pm 0.264$ & $46.421 \pm 0.691$ & $143 \pm 3$ \\
\hline Comp. 2 & $1.379 \pm 0.073$ & $169 \pm 2$ & $109720.494 \pm 0.600$ & $29.242 \pm 1.412$ & $47 \pm 3$ \\
\hline${ }^{12} \mathrm{C}^{17} \mathrm{OJ}=1 \rightarrow 0$ & $0.629 \pm 0.056$ & $286 \pm 5$ & $112252.191 \pm 1.894$ & $44.837 \pm 5.007$ & $14 \pm 2$ \\
\hline${ }^{13} \mathrm{C}^{16} \mathrm{O} J=2 \rightarrow 1$ & $123.010 \pm 0.261$ & $278 \pm 1$ & $220194.289 \pm 0.107$ & $105.105 \pm 0.272$ & $1170 \pm 4$ \\
\hline${ }^{12} \mathrm{C}^{18} \mathrm{O} J=2 \rightarrow 1$ & $33.905 \pm 0.212$ & $278 \pm 1$ & $219356.938 \pm 0.273$ & $92.815 \pm 0.734$ & $365 \pm 4$ \\
\hline${ }^{12} \mathrm{C}^{17} \mathrm{OJ}=2 \rightarrow 1$ & $4.216 \pm 0.147$ & $273 \pm 2$ & $224509.430 \pm 1.437$ & $86.434 \pm 3.724$ & $49 \pm 3$ \\
\hline \multicolumn{6}{|l|}{ Mrk 231} \\
\hline${ }^{12} \mathrm{C}^{16} \mathrm{O} J=1 \rightarrow 0$ & $4.687 \pm 0.034$ & $12658 \pm 01$ & $110601.313 \pm 00.268$ & $75.254 \pm 00.650$ & $2.3 \pm 0.7$ \\
\hline${ }^{13} \mathrm{C}^{16} \mathrm{O} J=1 \rightarrow 0$ & $0.178 \pm 0.041$ & $12723 \pm 28$ & $105714.712 \pm 09.762$ & $89.608 \pm 31.488$ & $2.0 \pm 0.8$ \\
\hline${ }^{12} \mathrm{C}^{18} \mathrm{OJ}=1 \rightarrow 0$ & $0.142 \pm 0.034$ & $12685 \pm 31$ & $105325.492 \pm 10.778$ & $75.964 \pm 26.302$ & $1.9 \pm 0.8$ \\
\hline${ }^{12} \mathrm{C}^{16} \mathrm{O} J=2 \rightarrow 1$ & $33.178 \pm 0.135$ & $12661 \pm 01$ & $221196.545 \pm 00.299$ & $151.903 \pm 00.731$ & $218.4 \pm 1.4$ \\
\hline${ }^{13} \mathrm{C}^{16} \mathrm{O} J=2 \rightarrow 1$ & $0.693 \pm 0.142$ & $12682 \pm 13$ & $211453.470 \pm 09.194$ & $91.493 \pm 21.645$ & $7.6 \pm 2.4$ \\
\hline${ }^{12} \mathrm{C}^{18} \mathrm{O} J=2 \rightarrow 1$ & $0.544 \pm 0.131$ & $12691 \pm 16$ & $210643.401 \pm 11.551$ & $73.979 \pm 22.168$ & $7.4 \pm 2.8$ \\
\hline $\mathrm{CN} N=1 \rightarrow 0$ & $0.295 \pm 0.053$ & - & $108590.836 \pm 06.522$ & $84.815 \pm 22.957$ & $3.5 \pm 1.1$ \\
\hline $\mathrm{CN} N=1 \rightarrow 0$ & $0.600 \pm 0.040$ & - & $108898.443 \pm 02.392$ & $73.780 \pm 05.832$ & $8.1 \pm 0.8$ \\
\hline $\mathrm{CN} N=2 \rightarrow 1$ & $1.714 \pm 0.189$ & - & $217691.713 \pm 07.215$ & $139.615 \pm 18.827$ & $12.3 \pm 2.1$ \\
\hline $\mathrm{CN} N=2 \rightarrow 1$ & $1.544 \pm 0.197$ & - & $217442.541 \pm 11.022$ & $177.146 \pm 25.067$ & $8.7 \pm 1.7$ \\
\hline $\mathrm{CN} N=2 \rightarrow 1$ & $0.499 \pm 0.117$ & - & $217165.557 \pm 08.030$ & $70.279 \pm 19.209$ & $7.1 \pm 2.6$ \\
\hline
\end{tabular}

Notes. Given frequencies refer to the local standard of rest (LSR) $V_{\mathrm{LSR}}=0 \mathrm{~km} \mathrm{~s}^{-1}$ frequency scale. All errors are standard deviations obtained from Gaussian fits, except those in the last column. The $T_{\mathrm{mb}}$ values and errors were derived by combining the values of Cols. 2 and 5 . Outflow components (see Turner et al. 1985; Feruglio et al. 2010; Aalto et al. 2012; Bolatto et al. 2013), not apparent in the weaker isotopic lines, are not included in the fits. For potential calibration errors, see Sect. 3.6.2. Because of the large bandwidths of the spectra, yielding potentially complex velocity-frequency correlations, frequencies and not velocities are emphasized. For the CO lines, however, optical (cz) $V_{\text {LSR }}$ values are also given, mainly to show the relative importance of the two main spectral features in NGC 253. Since each CN component represents a group of hyperfine components of different strengths, no velocities are given in this case.

low value in spite of all the corrections we have made. It is also small in view of the excitation temperatures derived from other species in NGC 253 (Martín et al. 2006), strongly indicating subthermal excitation. The critical density of $\mathrm{CN} N=1 \rightarrow 0$, where collisional excitation rates match those for spontaneous radiative decay, is high $\left(n_{\text {crit }} \sim 10^{6} \mathrm{~cm}^{-3}\right)$ and almost as large as that for $\mathrm{HCN} J=1 \rightarrow 0$. Therefore, subthermal emission from a predominantly lower density medium is no surprise. The corresponding CN column density is $1.7 \times 10^{15} \mathrm{~cm}^{-2}$ but could be up to a factor of 20 higher and almost an order of magnitude lower for the limiting cases $T_{\mathrm{ex}}=11.3$ and $3.0 \mathrm{~K}$ (the latter obtained prior to correct for $\mathrm{CN} N=2 \rightarrow 1$ line saturation). We further note that these column density estimates are speculative, because there may exist a hot, dense component with $T_{\mathrm{ex}}$ well above $10 \mathrm{~K}$, which might only become visible when observing higher $N$ transitions (for $\mathrm{CN}$ chemistry, see, e.g., Simon et al. 1997; Liszt \& Lucas 2001). Adopting exclusively collisional excitation and using RADEX (van der Tak et al. 2007), $T_{\mathrm{ex}}=4.0 \mathrm{~K}$ corresponds to a density of $n\left(\mathrm{H}_{2}\right) \sim 2.5 \times 10^{4} \mathrm{~cm}^{-3}$ for kinetic temperatures of $50-100 \mathrm{~K}$. This involves Einstein coefficients from Klisch et al. (1995), a dipole moment of 1.45 Debye
(Thomson \& Dalby 1968), and He-impact rates from Lique et al. (2010) scaled by 1.37 to simulate $\mathrm{H}_{2}$.

\subsection{Beyond CN: Oxygen isotope ratios in NGC 253}

Owing to bandwidths of $2 \times 2 \times 4 \mathrm{GHz}$ (Sect. 2), our spectra not only contain $\mathrm{CN}$ but also include a number of $\mathrm{CO}$ lines (Figs. 3-5 and Sect. 3.6.2). Because of their strength and because line shapes are reflecting individual components and not groups of hyperfine components (the exception to this rule is ${ }^{12} \mathrm{C}^{17} \mathrm{O}$, hereafter $\mathrm{C}^{17} \mathrm{O}$ ), not only is the $V_{\mathrm{LSR}} \sim 290 \mathrm{~km} \mathrm{~s}^{-1}$ feature seen in the $J=1 \rightarrow 0$ lines (see Sect. 3.2), but also the lower velocity $V_{\mathrm{LSR}} \sim 170 \mathrm{~km} \mathrm{~s}^{-1}$ component (e.g., Martín et al. 2006), which contributes $10 \%$ to $20 \%$ to the total line emission in our $\lambda=3 \mathrm{~mm}$ spectra. The $J=2 \rightarrow 1$ lines obtained with a smaller beam size (Table 1) are best fit by a single velocity component.

Table 3 summarizes the parameters of the Gaussian fits to the line profiles. Noteworthy are the extremely high $I\left(\mathrm{C}^{18} \mathrm{O}\right) / I\left(\mathrm{C}^{17} \mathrm{O}\right) J=1 \rightarrow 0$ and $2 \rightarrow 1$ ratios with respect to the Galactic interstellar medium ( 3.5; Wouterloot et al. 2008), 
$12.7 \pm 1.2$ and $8.0 \pm 0.3$. These have already been noted before (e.g., Henkel \& Mauersberger 1993), but the rotational lines of the different isotopologues could previously not be observed simultaneously. The high ratios of order 10 were interpreted in terms of vigorous massive star formation in a nuclear starburst, which contains a metal-rich gaseous composition.

Adopting ${ }^{12} \mathrm{C} /{ }^{13} \mathrm{C}=X=40 \pm 10$ from $\mathrm{CN}$ (Sect. 3.7) and keeping in mind that at least in Galactic star forming regions ${ }^{12} \mathrm{C} /{ }^{13} \mathrm{C}$ ratios from $\mathrm{CN}$ and $\mathrm{C}^{18} \mathrm{O}$ are similar (Milam et al. 2005), we can also estimate the optical depths of the various $\mathrm{CO}$ isotopologues. When only accounting for the errors obtained from Gaussian fits, the $\mathrm{CO} /{ }^{13} \mathrm{CO} J=1 \rightarrow 0$ line intensity ratio becomes $13.98 \pm 0.34$ (Table 3 ). Since the intensity of the CO $J=1 \rightarrow 0$ line may be overestimated by $30 \%$ (Sect. 3.6.2), we estimate a ratio of $R=10-14$ and obtain with

$R=\frac{1-\mathrm{e}^{-X \tau}}{1-\mathrm{e}^{-\tau}}$

where $\tau(\mathrm{CO} 1 \rightarrow 0)=X \tau=1.8-5$. This result implies that ${ }^{13} \mathrm{CO}$ should be optically thin, not only in the $J=1 \rightarrow 0$ but also in the $2 \rightarrow 1$ transition. Testing this by comparing the $I\left({ }^{13} \mathrm{CO}\right) / I\left(\mathrm{C}^{18} \mathrm{O}\right)$ line intensity ratios in the two ground rotational transitions, we obtain $3.601 \pm 0.046$ and $3.628 \pm 0.024$. Within the limits of accuracy, the ratios agree with each other, as expected in the case of optically thin emission. With the $I\left({ }^{13} \mathrm{CO}\right) / I\left(\mathrm{C}^{18} \mathrm{O}\right)$ value and with $8.9 \pm 1.2$ as the weighted mean of the $I\left(\mathrm{C}^{18} \mathrm{O}\right) / I\left(\mathrm{C}^{17} \mathrm{O}\right)$ ratio from the $J=1 \rightarrow 0$ and $2 \rightarrow$ 1 transitions, ${ }^{16} \mathrm{O} /{ }^{18} \mathrm{O}=\mathrm{CO} /{ }^{13} \mathrm{CO} \times{ }^{13} \mathrm{CO} / \mathrm{C}^{18} \mathrm{O}=(40 \pm 10) \times$ $(3.62 \pm 0.05)=145 \pm 36$ and ${ }^{16} \mathrm{O} /{ }^{17} \mathrm{O}={ }^{16} \mathrm{O} /{ }^{18} \mathrm{O} \times \mathrm{C}^{18} \mathrm{O} / \mathrm{C}^{17} \mathrm{O}=$ $(145 \pm 36) \times(8.9 \pm 1.2)=1290 \pm 365$ (see also Harrison et al. 1999).

\section{Other galaxies}

\section{1. $C N$ in ULIRGs}

In view of the extreme usefulness of $\mathrm{CN}$ as a tracer of the carbon isotope ratio (Sects. 3.3 and 3.6.1) and to complement our discussion of the relatively weak starburst in NGC 253, it is of interest to gain some idea of $N=1 \rightarrow 0$ and $2 \rightarrow 1 \mathrm{CN}$ emission from a truly luminous local ultraluminous infrared galaxy (ULIRG). With $L_{\mathrm{IR}} \sim 2.5 \times 10^{12} L_{\odot}$ at $z=0.0422$ (e.g., Aalto et al. 2012), Mrk 231 is the target of choice. The two lower panels of Fig. 6 show the spectra, and Table 3 displays line parameters.

With the enormous infrared luminosity of Mrk 231, hinting at a high mass of dense star forming molecular gas, one might naively expect that the $\mathrm{CN}$ lines should be more saturated than in NGC 253. However, this is not entirely the case. The two components of the $1 \rightarrow 0$ transition have relative intensities exactly as expected for optically thin lines under LTE conditions. Excluding the unlikely case that this is an unfortunate combination of intrinsic non-LTE line strengths and high optical depth, this provides support for our assumption (Sect. 3.7) that "intrinsic intensities" (i.e., intensities after removing saturation effects) follow LTE conditions also in NGC 253.

Nevertheless, the CN spectra from Mrk 231 appear to originate in a different environment from those from NGC 253. While the peak line temperatures of the $N=1 \rightarrow 0$ and $2 \rightarrow 1$ lines are similar toward NGC 253, the $2 \rightarrow 1$ features are significantly stronger than the $1 \rightarrow 0$ lines in Mrk 231. Furthermore, the integrated line intensity ratios of the three $2 \rightarrow 1$ components in Mrk 231 unambiguously indicate saturation. Instead of ratios of 1:5:9, expected in the optically thin case (Fig. 2), the integrated intensity ratios are roughly 1:3:3.5 (Fig. 6 and Table 3).
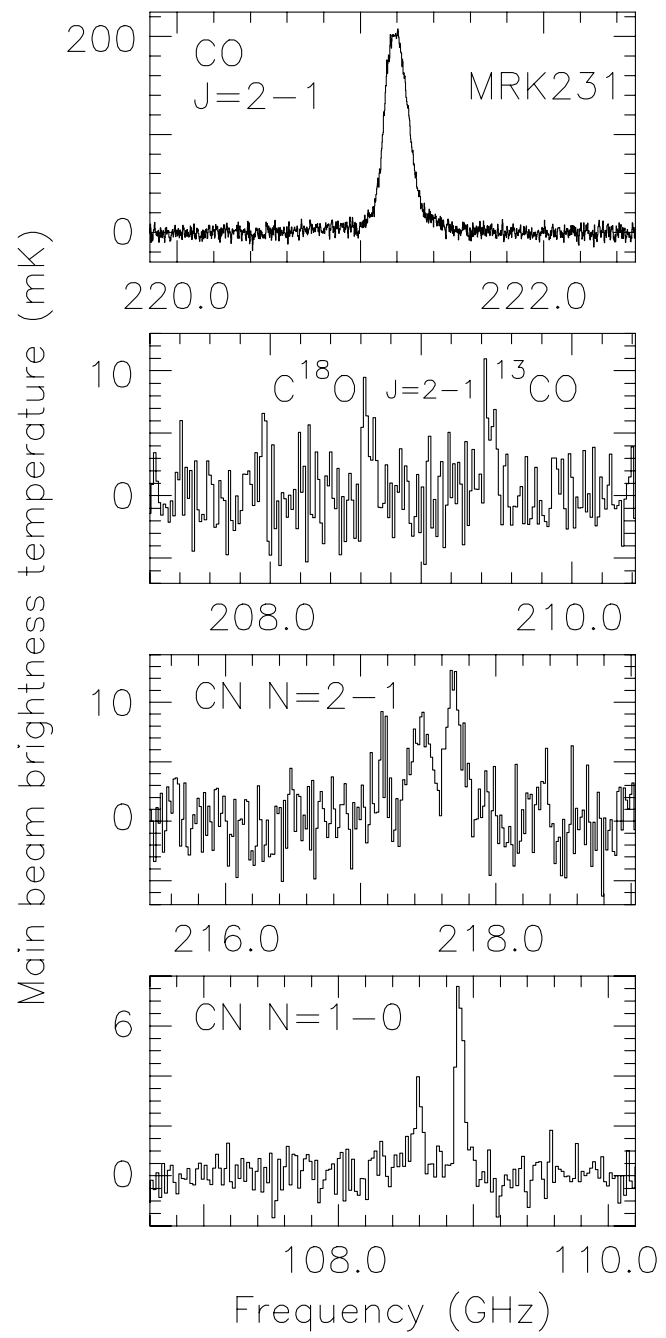

Fig. 6. $\mathrm{CO} J=2 \rightarrow 1$ and CN spectra from Mrk 231 on a LSR $V_{\mathrm{LSR}}=0 \mathrm{~km} \mathrm{~s}^{-1}$ frequency scale. The spectra were smoothed to channel spacings of $\sim 2.7,22,23$, and $60 \mathrm{~km} \mathrm{~s}^{-1}(\sim 2,16,16$, and $21 \mathrm{MHz})$ from top to bottom.

Therefore, it is clear that the $\mathrm{CN}$ excitation temperature must be higher in Mrk 231 than in NGC 253.

For a quantitative estimate, we assume again that the intrinsic intensities (i.e., intensities in the absence of line saturation) follow LTE conditions. (There is no indication of non-LTE effects, because features expected to be stronger are stronger and features expected to be weaker are indeed weaker; see Fig. 6.) Using the same approach as in Sect. 3.7, this yields opacities of $\tau=0.3 \pm 0.2,1.4_{-0.7}^{+1.2}$, and $2.9_{-0.9}^{+1.5}$ for the three $\mathrm{CN}$ $N=2 \rightarrow 1$ components. To correct relative column densities for these optical depths by $\tau /\left(1-\mathrm{e}^{-\tau}\right)$, we obtain a factor of $2.3_{-0.6}^{+1.0}$, yielding a modified intensity ratio of $I^{\prime}(\mathrm{CN} 2 \rightarrow 1) / I(\mathrm{CN}$ $1 \rightarrow 0)=9.73_{-2.31}^{+4.35}$. Since we integrated over frequency and not yet over velocity, this value has to be reduced by a factor of two. Accounting for the different beam sizes at $\lambda=3$ and $1.3 \mathrm{~mm}$ and realizing that Mrk 231 is spatially unresolved in all of the 30-m beams (e.g., Aalto et al. 2012), the final ratio is $I(\mathrm{CN} 2 \rightarrow$ $1) / I(\mathrm{CN} 1 \rightarrow 0)=1.22_{-0.29}^{+0.54}$. By adopting the procedure outlined in Sect. 3.5, this yields an excitation temperature of $T_{\mathrm{ex}}=$ $8.4_{-1.6}^{+3.8} \mathrm{~K}$ and a tentative $22^{\prime \prime}$ beam-averaged column density of $N(\mathrm{CN}) \sim 3.4_{-1.7}^{+7.4} \times 10^{14} \mathrm{~cm}^{-2}$. Using RADEX (see Sect. 3.7), the corresponding density becomes $n\left(\mathrm{H}_{2}\right) \sim 8 \times 10^{4} \mathrm{~cm}^{-3}$. While uncertainties are large, this hints at an excitation temperature that 
is about twice as large as in NGC 253 (Sect. 3.5) and NGC 4945 (Wang et al. 2004) and indicates that $\mathrm{CN}$ transitions with quantum numbers $N>2$ may be of interest, at least in ULIRGs.

\subsection{The carbon isotope ratio in starbursts across the universe}

As we have seen (Sect. 3.7), the ${ }^{12} \mathrm{C} /{ }^{13} \mathrm{C}$ ratio of the nuclear starburst in NGC 253 is, at least for the high velocity ( $V_{\mathrm{LSR}} \sim$ $290 \mathrm{~km} \mathrm{~s}^{-1}$; Sect. 3.2) component, higher than in our Galactic center region. The presence of a bar (e.g., Engelbracht et al. 1998), likely providing significant inflow, may be a crucial factor that directs large quantities of fresh and poorly proccessed gas toward the nuclear region of NGC 253. While this gas also reaches the central part of our Galaxy (Riquelme et al. 2010), this process may occur on a much larger scale in NGC 253, where even indications of massive molecular feed back have already been detected (Turner 1985; Bolatto et al. 2013). For ongoing star formation, inflowing gas with high ${ }^{12} \mathrm{C} /{ }^{13} \mathrm{C}$ ratios may then become even more enriched in ${ }^{12} \mathrm{C}$ by the material ejected from young massive stars.

Are all starburst galaxies alike with respect to their carbon isotope ratio? Here we may differentiate between starbursts in their early and late stages of evolution, as well as between weak and strong starbursts (see, e.g., Fig. 1 in Mao et al. 2010), the latter leading to the presence of (ultra)luminous infrared galaxies ((U)LIRGs). Finally, we may also distinguish between galaxies in the local and in the early universe.

NGC 253 has been believed to host a young starburst (e.g., García-Burillo et al. 2000; Wang et al. 2004), but in view of detected large scale outflows (Turner 1985; Bolatto et al. 2013), an intermediate stage of evolution appears to be more likely. With a total infrared luminosity of $L_{\mathrm{IR}} \sim 3 \times 10^{10} L_{\odot}$ (e.g., Henkel et al. 1986), its level of activity is at the low end of the range observed in the starbursts of spiral galaxies. A comparison of the carbon isotope ratio in NGC 253 with a starburst in a late stage of evolution of similar infrared luminosity, M 82, is not yet possible. Henkel et al. (1998) studied M 82 and proposed an isotope ratio of ${ }^{12} \mathrm{C} /{ }^{13} \mathrm{C}>40$ based on $\mathrm{CN}$, and Martín et al. (2010) report a value $>138$ from $\mathrm{C}_{2} \mathrm{H}$, but both results should be taken with some degree of scepticism, because ${ }^{13} \mathrm{CN}$ and ${ }^{13} \mathrm{CCH}$ or $\mathrm{C}^{13} \mathrm{CH}$ were not detected.

Mrk 231, one of the most luminous galaxies within a billion lightyears from Earth, contains an active galactic nucleus (AGN) and may host a starburst in a late stage of evolution. This can be deduced from the presence of only one nucleus in this galaxy merger and intense outflows of ionized and molecular gas (e.g., Feruglio et al. 2010; Aalto et al. 2012), rapidly exhausting the molecular star forming fuel in the central region. Figures 3, 4, and 6 show $\mathrm{CO},{ }^{13} \mathrm{CO}$, and $\mathrm{C}^{18} \mathrm{O}$ profiles. Comparing NGC 253 with Mrk 231, we note that the $\mathrm{CO}$ and $\mathrm{C}^{18} \mathrm{O} J=1 \rightarrow 0$ peak temperatures are $\sim 100$ times higher in NGC 253 , while for ${ }^{13} \mathrm{CO}$ $J=1 \rightarrow 0$, the ratio exceeds 200 . The $I\left({ }^{12} \mathrm{CO}\right) / I\left(\mathrm{C}^{18} \mathrm{O}\right)$ values are $30-40$ in both sources. However, the ${ }^{12} \mathrm{CO} /{ }^{13} \mathrm{CO}$ line ratios are quite different, with $\left.I\left({ }^{12} \mathrm{CO}\right) / I\left({ }^{13} \mathrm{CO}\right)\right)=10-14$ in NGC 253 (Sect. 3.8) and 25-50 in Mrk 231 (Table 3). While the ${ }^{12} \mathrm{C} /{ }^{13} \mathrm{C}$ ratio in the nuclear region of NGC 253 is already higher than in our Galactic center region, it appears to be even higher in Mrk 231. In NGC 253, ${ }^{13} \mathrm{CO}$ is much stronger than $\mathrm{C}^{18} \mathrm{O}$ in both ground rotational lines. In Mrk 231 both lines show similar intensities (Figs. 3 and 6) and should be optically thin in view of their weakness relative to $\mathrm{CO}$.

Overall, assuming that the $\mathrm{CO} / \mathrm{C}^{18} \mathrm{O}$ abundance ratios are the same, Mrk 231 should have a deficit in ${ }^{13} \mathrm{C}$ by a factor of almost
Table 4. Extragalactic carbon isotope ratios.

\begin{tabular}{ccrc}
\hline \hline Class & Target & ${ }^{12} \mathrm{C} /{ }^{13} \mathrm{C}$ & Ref. \\
\hline Quiescent spiral, center & Milky Way & $\sim 25$ & 1 \\
Low level starburst & NGC 253 & $\sim 40$ & 2 \\
Local ULIRGs & Mrk 231/Arp 220 & $\sim 100$ & 2,3 \\
Redshift $z=2.5$ ULIRG & Cloverleaf & $>100$ & 4 \\
\hline
\end{tabular}

References. (1) Güsten et al. (1985); (2) this paper; (3) González-Alfonso et al. (2012); (4) Henkel et al. (2010).

three relative to NGC 253 (for a statistical evaluation comprising many galaxies, see Taniguchi \& Ohyama 1998; Taniguchi et al. 1999), possibly yielding ${ }^{12} \mathrm{C} /{ }^{13} \mathrm{C} \sim 100$ and thus also ${ }^{16} \mathrm{O} /{ }^{18} \mathrm{O} \sim$ 100. Interestingly, Greve et al. (2009) and Martín et al. (2011) also find $I\left({ }^{13} \mathrm{CO}\right) \sim I\left(\mathrm{C}^{18} \mathrm{O}\right)$ for the less evolved merger Arp 220. Furthermore, González-Alfonso et al. (2012) derive ${ }^{16} \mathrm{O} /{ }^{18} \mathrm{O} \sim$ 100 from $\mathrm{OH}$ Herschel data for the same source. Arp 220 still possesses two well separated nuclei and does not yet show any outflow that could match what is seen in Mrk 231. In view of their different stages of evolution, it is therefore surprising that Arp 220 and Mrk 231 can be characterized by quite similar carbon and ${ }^{16} \mathrm{O} /{ }^{18} \mathrm{O}$ ratios. Even the utraluminous eyelash galaxy at redshift 2.3, the first high- $z$ galaxy with detected $\mathrm{C}^{18} \mathrm{O}$ emission, shows similar ${ }^{13} \mathrm{CO}$ and $\mathrm{C}^{18} \mathrm{O}$ intensities (Danielson et al. 2013), indicating a ${ }^{13} \mathrm{C}$ depletion with respect to local more quiescent galaxies. The LIRG NGC 1068 with an uncorrected $I\left({ }^{12} \mathrm{CN}\right) / I\left({ }^{13} \mathrm{CN}\right)$ ratio of $\sim 50$ (Aladro et al. 2013) might be an intermediate case (however, its ${ }^{13} \mathrm{CN}$ features are weaker and therefore show lower signal-to-noise ratios than those displayed in our Fig. 1). The Cloverleaf quasar at redshift $z \sim 2.5$ appears to be even more extreme. Based on a large number of $\mathrm{CO}$ data and the first detection of ${ }^{13} \mathrm{CO}$ at high redshift, the ${ }^{12} \mathrm{C} /{ }^{13} \mathrm{C}$ ratio should be well above 100 (Henkel et al. 2010).

A summary of these results is given in Table 4, where sources with different properties are listed with their carbon isotope ratio. This is a first attempt to set up such a table. In the Galaxy, there is not only a carbon isotope ratio gradient, but also indications of dispersion at a given galactocentric radius (e.g., Milam et al. 2005), which is expected in view of radial gas streaming and potential cloud-to-cloud variations due to local supernovae or ejecta by late-type stars. With respect to external galaxies, we are still far away from such a level of precision. More accurate determinations of the carbon isotope ratio in the galaxies listed in Table 4, as well as in other extragalactic targets, including other classes of objects, are urgently needed. An obvious example would be M 82 as the prototype for a weak starburst at a late stage of evolution. Submillimeter galaxies (SMGs) would also be attractive.

The few determined values (Table 4 ) indicate a trend with high- $z$ ULIRGs showing the highest carbon isotope ratios. Low- $z$ ULIRGs appear to contain more processed material but still show values near ${ }^{12} \mathrm{C} /{ }^{13} \mathrm{C} \sim 100$. The high values compared to those found in most parts of the Milky Way indicate that (1) the bulk of the material originates in a massive inflow of poorly processed ${ }^{13} \mathrm{C}$ deficient gas and/or that (2) there is a large input of ${ }^{12} \mathrm{C}$-rich gas from ejecta of massive stars. The latter might become more dominant, if the number of such stars were enhanced by a top-heavy stellar initial mass function, a result of the high kinetic temperatures expected in extreme cosmic-ray-dominated environments (Papadopoulos et al. 2011). Weaker starbursts may show a moderate enhancement over the classical value for the Galactic center region, but all these statements are still based on 
a rather small number of sources. Right now, we are only beginning to collect the required data for a comprehensive understanding of $\mathrm{CNO}$ and $\mathrm{S} / \mathrm{Si}$ nucleosynthesis based on extragalactic molecular spectra.

\section{Conclusions}

Using the IRAM 30-m telescope at Pico Veleta, we have detected two $\mathrm{CN}$ isotopologues toward the nearby starburst galaxy NGC 253, four CO isotopologues toward NGC 253, and three $\mathrm{CO}$ isotopologues toward the ultraluminous merger galaxy Mrk 231. We also presented CN $N=1 \rightarrow 0$ and $2 \rightarrow 1$ spectra from Mrk 231. The main results of this study are:

- $\mathrm{CN}$ appears to be the best tracer to determine carbon isotope ratios in nearby external galaxies.

- Toward NGC 253, the measured ${ }^{13} \mathrm{CN} N=1 \rightarrow 0$ line intensities are compatible with local thermodynamical equilbrium (LTE) under optically thin conditions. The relative line intensities of the ${ }^{12} \mathrm{CN} N=1 \rightarrow 0$ features are best explained by LTE conditions modified by moderate saturation, affecting the peak intensity of the weaker component by $\sim 10 \%$ and the stronger component by $\sim 25 \%$. For ${ }^{12} \mathrm{CN} 2 \rightarrow 1$, either the weakest of the three observed line components is enhanced or the feature of intermediate intensity is depleted relative to the expected LTE intensity under optically thin conditions.

- Accounting for calibration uncertainties and moderate saturation in the ${ }^{12} \mathrm{CN} 1 \rightarrow 0$ line, the ${ }^{12} \mathrm{C} /{ }^{13} \mathrm{C}$ isotope ratio becomes $40 \pm 10$ for the molecular core peaking some arcseconds southwest of the dynamical center of NGC 253. Combined with data from several $\mathrm{CO}$ isotopologues and adopting this ${ }^{12} \mathrm{C} /{ }^{13} \mathrm{C}$ ratio also for the $\mathrm{CO}$ emitting gas (which is supported by results from Galactic $\mathrm{CN}$ and $\mathrm{C}^{18} \mathrm{O}$ data), this yields ${ }^{16} \mathrm{O} /{ }^{18} \mathrm{O}=145 \pm 36$ and ${ }^{16} \mathrm{O} /{ }^{17} \mathrm{O}=$ $1290 \pm 365$.

- Both $\mathrm{CN}$ and $\mathrm{C}_{2} \mathrm{H}$ show a number of hyperfine components, which allows us to determine optical depths even in extragalactic spectra covering a broad velocity range. A systematic survey of $\mathrm{C}_{2} \mathrm{H}$ and its ${ }^{13} \mathrm{C}$-bearing isotopologues in star forming clouds of the Galaxy would thus be essential to check whether resulting carbon isotope ratios are consistent with those already derived from $\mathrm{H}_{2} \mathrm{CO}, \mathrm{C}^{18} \mathrm{O}$, and $\mathrm{CN}$.

- Toward NGC 253, there is no indication of vibrationally excited $\mathrm{CN}$. The lower frequency fine structure components in the $v=1, N=1 \rightarrow 0$, and $2 \rightarrow 1$ transitions are not seen down to rms levels of 3 and $4 \mathrm{mK}$ (15 and $20 \mathrm{mJy}$ ) in $8.5 \mathrm{~km} \mathrm{~s}^{-1}$ wide channels. Those at higher frequency are blended by $\mathrm{C}^{17} \mathrm{O}$.

- The CN excitation temperature in NGC 253, derived from the $N=1 \rightarrow 0$ and $2 \rightarrow 1$ lines, is $3-11 \mathrm{~K}$, with a most likely value of $T_{\mathrm{ex}} \sim 4 \mathrm{~K}$. With this value, the column density becomes $N(\mathrm{CN})=2 \times 10^{15} \mathrm{~cm}^{-2}$ and the density, assuming purely collisional excitation, becomes $n\left(\mathrm{H}_{2}\right) \sim$ $2.5 \times 10^{4} \mathrm{~cm}^{-3}$.

- CN data from the ultraluminous merger Mrk 231 indicate that the excitation temperature is enhanced by a factor of two with respect to NGC 253 and NGC 4945. In Mrk 231, relative $\mathrm{CN}$ line intensities within the $N=1 \rightarrow 0$ and $2 \rightarrow 1$ transitions are compatible with local thermodynamical equilibrium. While the $1 \rightarrow 0$ transitions appear to be optically thin, the $2 \rightarrow 1$ lines show significant saturation effects. In view of the excitation temperature, which indicates a density of almost $10^{5} \mathrm{~cm}^{-3}$ assuming exclusively collisional excitation, it would make sense to observe $\mathrm{CN}$ transitions with higher quantum numbers $N$ in Mrk 231 and other ultraluminous infrared galaxies (ULIRGs).

- A comparison between NGC 253 and Mrk 231 shows that ${ }^{13} \mathrm{C}^{16} \mathrm{O}$ is underabundant in Mrk 231 relative to ${ }^{12} \mathrm{C}^{16} \mathrm{O}$ and ${ }^{12} \mathrm{C}^{18} \mathrm{O}$ by almost a factor of three. This would yield ${ }^{12} \mathrm{C} /{ }^{13} \mathrm{C}$ $\sim 100$ and, because ${ }^{13} \mathrm{CO}$ and $\mathrm{C}^{18} \mathrm{O}$ show similar intensities in both the $J=1 \rightarrow 0$ and $2 \rightarrow 1$ lines, also ${ }^{16} \mathrm{O} /{ }^{18} \mathrm{O} \sim$ 100. This is similar to the values determined for Arp 220, even though Arp 220 is a much less evolved ultraluminous merger.

- Obtaining a synthesis of the carbon isotope ratios obtained so far from the central regions of actively star forming galaxies, the observed range of values appears to encompass a full order of magnitude. From ultraluminous galaxies at high redshift to local ULIRGs, to weaker local starbursting galaxies, and to the central molecular zone of the Milky Way, ratios are $>100, \sim 100, \sim 40$, and 25 , respectively. While this matches qualitative expectations of decreasing ${ }^{12} \mathrm{C} /{ }^{13} \mathrm{C}$ values with time and metallicity, we note that (1) the extragalactic values are based on an extremely small data base and that (2) the ratios for the ULIRGs at high and low $z$ are still rather uncertain. Furthermore, it still has to be evaluated how much ${ }^{13} \mathrm{C}$-deficient gas from the outer galactic regions and ${ }^{12} \mathrm{C}$-rich ejecta from massive stars in a nuclear starburst (the latter possibly enhanced by a top-heavy initial mass function) are contributing to raise the carbon isotope ratios during the lifetime of a starburst.

Acknowledgements. We wish to thank the IRAM staff at the 30-m for their help with the observations and C. M. Walmsley and an anonymous referee for carefully reading the manuscript. Some of the work by C.H. has been carried out while visiting the ESO-ALMA group in Santiago de Chile. A.L.R.D. acknowledges an STFC studentship (ST/F007299/1).

\section{References}

Aalto, S., Black, J. H., Johansson, L. E. B., \& Booth, R. S. 1991, A\&A, 249, 323 Aalto, S., García-Burillo, S., Muller, S., et al. 2012, A\&A, 537, A44 Abia, C., Palmerini, S., Busso, M., \& Cristallo, S. 2012, A\&A, 548, A55 Aladro, R., Viti, S., Bayet, E., et al. 2013, A\&A, 549, A39

Bogey, M., Demuynck, C., \& Destombes, J. L. 1984, Can. J. Phys., 62, 1248 Bolatto, A. D., Warren, S. R., Leroy, A. K., et al. 2013, Nature, 499, 450 Casoli, F., Dupraz, C., \& Combes, F. 1992, A\&A, 264, 55

Chiappini, C., Matteucci, F., \& Romano, D. 2001, ApJ, 554, 1044

Chin, Y.-N., Henkel, C., Whiteoak, J. B., Langer, N., \& Churchwell, E. B. 1996, A\&A, 305, 960

Danielson, A. L. R., Swinbank, A. M., Smail, I., et al. 2013, MNRAS, 436, 2793 Engelbracht, C. W., Rieke, M. J., Rieke, G. H., Kelly, D. M., \& Achtermann, J. M. 1998, ApJ, 505, 639

Feruglio, C., Maiolino, R., Piconcelli, E., et al. 2010, A\&A, 518, L155

García-Burillo, S., Martín-Pintado, J., Fuente, A., \& Neri, R. 2000, A\&A, 355, 499

Gerin, M., Combes, F., Encrenaz, P., et al. 1984, A\&A, 136, L17

González-Alfonso, E., Fischer, J., Graciá-Carpio, et al. 2012, A\&A, 541, A4

Greve, T. R., Papadopoulos, P. P., Gao, Y., \& Radford, S. J. E. 2009, ApJ, 692, 1432

Güsten, R., Henkel, C., \& Batrla, W. 1985, A\&A, 149, 195

Güsten, R., Philipp, S. D., Weiß, A., \& Klein, B. 2006, A\&A, 454, L115

Harrison, A., Henkel, C., \& Russel, A. 1999, MNRAS, 303, 157

Henkel, C., \& Mauersberger, R. 1993, A\&A, 274, 730

Henkel, C., Wilson, T. L., \& Bieging, J. 1982, A\&A, 109, 344

Henkel, C., Güsten, R., \& Gardner, F. F. 1985, A\&A, 143, 148

Henkel, C., Wouterloot, J. G. A., \& Bally, J. 1986, A\&A, 155, 193

Henkel, C., Mauersberger, R., \& Schilke, P. 1988, A\&A, 201, L23

Henkel, C., Mauersberger, R., Wiklind, T., et al. 1993, A\&A, 268, L17

Henkel, C., Wilson, T. L., Langer, N., Chin, Y.-N., \& Mauersberger, R. 1994, in The Stucture and Content of Molecular Clouds, Lect. Notes Phys., 439, 72

Henkel, C., Chin, Y.-N., Mauersberger, R., \& Whiteoak, J. B. 1998, A\&A, 329, 443

Henkel, C., Tarchi, A., Menten, K. M., \& Peck, A. B. 2004, A\&A, 414, 117

Henkel, C., Downes, D., Weiß, A., Riechers, D., \& Walter, F. 2010, A\&A, 516, A111 
Hodge, P. 1989, ARA\&A, 27, 139

Klisch, E., Klaus, T., Belev, S. P., Winnewisser, G., \& Herbst, E. 1995, A\&A, 304, L5

Langer, W. D., \& Penzias, A. A. 1990, ApJ, 357, 477

Langer, W. D., Graedel, T. E., Frerking, M. A., \& Armentrout, P. B. 1984, ApJ, 277, 581

Lebrón, M., Mangum, J. G., Mauersberger, R., et al. 2011, A\&A, 534, A56

Levshakov, S. A., Centurión, M., Molaro, P., \& Kostina, M. V. 2006, A\&A, 447, L21

Lique, F., Spielfiedel, A., Feautrier, N., et al. 2010, J. Chem. Phys., 132, 024303 Liszt, H., \& Lucas, R. 2001, A\&A, 370, 576

Mao, R.-Q., Schulz, A., Henkel, C., et al. 2010, ApJ, 724, 1336

Martín, S., Martín-Pintado, J., Mauersberger, T., Henkel, C., \& García-Burillo, S. 2005, ApJ, 620, 210

Martín, S., Mauersberger, R., Martín-Pintado, J., Henkel, C., \& García-Burillo, S. 2006, ApJS, 164, 450

Martín, S., Aladro, R., Martín-Pintado, \& Mauersberger, R. 2010, A\&A, 522, A62

Martín, S., Krips, M., Martín-Pintado, J., et al. 2011, A\&A, 527, A36

Martín, S., Martín-Pintado, J., Montero-Castaño, Ho, P. T. P., \& Blundell, R. 2012, A\&A, 539, A29

Mauersberger, R., Henkel, C., Wielebinski, R., Wiklind, T., \& Reuter, H.-P. 1996, A\&A, 305, 421

Mikolaitis, Ŝ, Tautvaiŝiené, G., Gratton, R., Bragaglia, A., \& Carretta, E. 2012, A\&A, 541, A137

Milam, S. N., Savage, C., Brewster, M. A., Ziurys, L. M., \& Wyckoff, S. 2005, ApJ, 634, 1126

Mouhcine, M., Ferguson, H. C., Rich, R. M., Brown, T. M., \& Smith, T. E. 2005, ApJ, 633, 810

Muller, S., Guélin, M., Dumke, M., Lucas, R., \& Combes, F. 2006, A\&A, 458, 417

Padovani, M., Walmsley, C. M., Tafalla, M., Galli, D., \& Müller, H. S. P. 2009, A\&A, 505, 1199
Paglione, T. A. D., Yam, O., Tosaki, T., \& Jackson, J. M. 2004, ApJ, 611, 835 Papadopoulos, P. P., Thi, W.-F., Miniati, F., \& Viti, S. 2011, MNRAS, 414, 1705 Pence, W. D. 1981, ApJ, 247, 473

Peng, R., Zhou, S., Whiteoak, J. B., Lo, K. Y., \& Sutton, E. C. 1996, ApJ, 470, 821

Penzias, A. A. 1980, Science, 208, 663

Puche, D., Carignan, C., \& van Gorkom, J. H., 1991, AJ, 101, 456

Rekola, R., Richer, M. G., McCall, M. L., et al. 2005, MNRAS, 361, 330

Rimmer, P. B., Herbst, E., Morata, O., \& Roueff, E. 2012, A\&A, 537, A7

Riquelme, D., Amo-Baladrón, M. A., Martín-Pintado, J., et al. 2010, A\&A, 523, A51

Sakamoto, K., Mao, R.-Q., Matsushita, S., et al. 2011, ApJ, 735, 19

Sheffer, Y., Rogers, M., Federman, S. R., Lambert, D. L., \& Gredel, R. 2007, ApJ, 667, 1002

Simon, R., Stutzki, J., Sternberg, A., \& Winnewisser, G. 1997, A\&A, 327, L9

Skatrud, D. D., De Lucia, F. C., Blake, G. A., \& Sastry, K. V. L. N. 1983, J. Mol. Spectr., 99, 35

Spielfiedel, A., Feautrier, N., Najar, F., et al. 2012, MNRAS, 421, 1891

Stahl, O., Wilson, T. L., Henkel, C., \& Appenzeller, I. 1989, A\&A, 221, 321

Taniguchi, Y., \& Ohyama, Y. 1998, ApJ, 507, L121

Taniguchi, Y., Ohyama, Y., \& Sanders, D. B. 1999, ApJ, 522, 214

Thomson, R., \& Dalby, F. W. 1968, Can. J. Phys., 46, 2815

Turner, B. E. 1985, ApJ, 299, 312

Ulvestad, J. S., \& Antonucci, R. R. J. 1997, ApJ, 488, 621

van der Tak, F. F. S., Black, J. H., Schöier, F. L., Jansen, D. J., \& van Dishoeck, E. F. 2007, A\&A, 468, 627

Wang, M., Henkel, C., Chin, Y.-N., et al. 2004, A\&A, 422, 883

Wang, M., Chin, Y.-N., Henkel, C., Whiteoak, J. B., \& Cunnningham, M. 2009, ApJ, 690, 580

Wannier, P. G. 1980, ARA\&A, 18, 399

Wilson, T. L., \& Rood, R. 1994, ARA\&A, 32, 191

Wouterloot, J. G. A., \& Brand, J. 1996, A\&AS, 119, 439

Wouterloot, J. G. A., Henkel, C., Brand, J., \& Davis, G. R. 2008, A\&A, 487, 237 